\title{
Decaying states of plane strain in a semi-infinite strip and boundary conditions for plate theory +
}

\author{
R. DOUGLAS GREGORY * and FREDERIC Y.M. WAN ** \\ * Department of Mathematics, University of Manchester, Manchester, Ml3 9PL, England \\ ** Department of Mathematics and Institute of Applied Mathematics and Statistics, The University of British \\ Columbia, Vancouver, B.C. V6T 1 Y4, Canada
}

(Received February 22, 1982)

\begin{abstract}
Friedrichs and Dressler and Gol'denveiser and Kolos have independently shown that the classical plate theory of Kirchhoff is the leading term of the outer expansion solution (in a small thickness parameter) for the linear elasto-statics of thin, flat, isotropic bodies. As expected, neither this leading term nor the full outer solution alone is able to satisfy arbitrarily prescribed edge conditions. On the other hand, the inner solution, which is significant only near the edge, is determined by a sequence of boundary value problems which are very difficult to solve, nearly as difficult as the original problem. For stress edge-data, St. Venant's principle may be invoked to generate a set of stress boundary conditions for the classical plate theory as well as for some higher order terms in the outer expansion without any reference to the inner solution. Attempts in the literature to derive the corresponding boundary conditions for displacement edge-data have not been successful.

With the help of the Betti-Rayleigh reciprocity theorem, we have derived the correct set of boundary conditions for classical and higher order plate theories with arbitrary edge-data. In this paper, we work out these conditions for an infinite plate strip with edgewise uniform data. We show that the conditions for individual terms in the outer expansion may be summed to give a simple set of appropriate boundary conditions for the full outer solution at the mid -plane. The boundary conditions obtained for the semi-infinite plate case are rigorously correct and the result for the stress data case rigorously justifies the application of

St. Venant's principle. Applications of the displacement boundary conditions obtained are illustrated by two simple problems: (i) The shearing of an infinitely long rectangular block, and (ii) A clamped infinite plate strip under uniform face pressure.
\end{abstract}

\section{Abklingende ebene Dehnungszustände in einem halb-unendlichen Streifen und $R$ andbe- dingungen fur die Plattentheorie (Z usammenfassung)}

Friedrichs und Dressler sowie Gol'denveiser und Kolos haben unabhängig voneinander gezeigt, dass das erste Glied der durch äussere Entwicklung (nach einem kleinen Dickeparamter) gewonnenen Lösung für die lineare Elastostatik dünner ebener, isotroper Körper zur klassischen Kirchhoffschen Plattentheorie führt. W i e erwartet kann weder dieses erste Glied noch die vollständige äussere Lösung allein willkürlich vorgegebene Randwerte erfüllen. Andererseits ist die innere Lösung die nur in Randnähe von Bedeutung ist, durch eine Folge von Randwertproblemen bestimmt, die sehr schwer zu lösen sind, nahezu ebenso schwer wie

+ The research is partly supported by NSERC Operating Grant No. A9259 and, in the case of the second author, also by a UBC Killam Senior Fellowship. 
das ursprüngliche Problem. Im Fall von Randbedingungen für die Spannungen kann man das St. Venantsche Prinzip sowohl zur Erzeugung eines Systems von Spannungsrandbedingungen für die klassische Plattentheorie als auch für einige Glieder höherer Ordnung in der äusseren Entwicklung ohne jeden Bezug zur inneren Lösung heranziehen. In der Literatur dargestellte Versuche, die entsprechenden Bedingungen fiir das Verschiebungsrandwertproblem herzuleiten, waren nicht erfolgreich.

Mit Hilfe des Betti-Rayleighschen Reziprozitätssatzes haben wir das korrekte System von Randbedingungen für die klassische Plattentheorie und auch für Plattentheorien höherer Ordnung mit willktirlichen Randwerten hergeleitet. In der vorliegenden Arbeit stellen wir diese Bedingungen für einen unendlichen Plattenstreifen mit gleichmässigen Randwerten auf. Wir zeigen, dass man durch Aufsummieren der Bedingungen für die einzelnen Glieder der äusseren entwicklung ein einfaches System angemessener Randbedingungen für die vollständige äussere Lösung ander Mittelebene erhalten kann. Die Randbedingungen, die man für den Fall einer half-unendlichen Platte erhält sind streng gültig und das Ergebnis fiir den Fall von vorgegebenen Spannungen rechtfertigt die Anwendung des St. Venantschen Prinzips vollkommen. Zwei einfache Probleme illustrieren die Anwendung der für das Verschiebungsproblem erhaltenen Randbedingungen: (i) Die Scherung eines unendlich langen rechteckigen Blocks und (ii) Ein eingespannter unendlicher Plattenstreifen unter gleichmässiger Belastung.

\section{Introduction}

It has been known since the work of Friedrichs and Dressler [1-3] that the classical Kirchhoff plate theory [4] corresponds to the leading term of the "interior (outer) expansion" (in powers of a thickness parameter $\varepsilon$ ) of the exact ${ }^{1}$ theory for the plate. For $\varepsilon \ll 1$, this leading term solution is expected to be a good approximation to the exact solution away from the edge of the plate. In general, this interior expansion is to be complemented by an "edge zone (inner) expansion" which gives the correct plate behaviour adjacent to an edge. The governing differential equations and boundary conditions for successive terms of these two asymptotic expansions are derived in $[1,3]$. Simplified versions of the edge zone expansion of [1,3] and the matching of interior and edge zone solutions within the framework of outer-inner expansions are given by Reiss and Locke [5] and by Reiss [6].

The parametric expansion methods of solution of $[1,3,5,6]$ all require a joint consideration of the two types of expansions to determine the interior expansion beyond its leading term. The same is true for the method of iterations used by Gol'denveiser and Kolos in [7,8] to get the "basic" state of stress (which is significant throughout the plate and is effectively the interior solution mentioned above) and the "boundary layer" states (which are significant only near the plate edge and are therefore edge zone corrections). Certain "damping conditions" for the boundary layer states, derived by invoking the St. Venant principle in [8], were later used by Kolos in [9] to eliminate the explicit appearance of the boundary layer states from the boundary conditions for the successive iterates of the basic state. The effect of the boundary layer states appears in the resulting boundary conditions only through integrals (across the plate thickness) of the solution of a number of canonical auxiliary problems for semi-infinite strips, evaluated at the strip end. Unfortunately, this rather novel approach to a difficult problem falls short of its promise for a number of reasons. The

1 By the exact theory we mean the three-dimensional, linear theory of elastostatics, for homogeneous, isotropic materials. 
most important one is that some of the canonical problems needed for obtaining displacement boundary conditions for successive iterates of the basic state are ill-posed and do not have a solution (as we shall show in section (4) of this paper). in addition, the procedure for constructing all boundary conditions for iterates of the basic state is extremely complicated and tedious, so that we cannot reasonably expect to go beyond $\mathrm{O}(\mathrm{E})$ correction terms in the interior solution. (Such a two-term approximate interior solution for an infinite plate with a circular hole has been obtained by van der Heijden in [10] where an earlier paper of the same type by Reiss [11] is also discussed.)

Earlier, E. Reissner applied a variational principle to an outer expansion for plate bending to get a set of stress boundary conditions for successive terms of the expansion [12]. (A detailed account of the historical developments of refining classical plate theory can also be found there.) The fact that deviations from the successive correction terms in the interior expansion gave rise to only self-equilibrating edge stresses in this case provided a natural setting for invoking the St. Venant principle to infer the rapid decay of the deviations from the interior solution [12]. Although Kolos did not actually work out his proposed boundary conditions for the interior solution in the case of plate bending under general edge tractions, it is clear from the form of his damping conditions that these stress boundary conditions generally do not agree with those obtained by Reissner. They do agree in the case of a straight edge with prescribed tractions uniform along the edge.

The corresponding boundary conditions for the interior solution have not been obtained in the literature for plates with general edge displacement data, though special cases have been worked out in [9] with a number of constants still to be determined from the solution of ill-posed semi-infinite strip problems. Beyond the nonexistence of the solutions for the canonical strip problems needed for these special results, there is also the question whether St. Venant's principle is sufficient to guarantee the "boundary layer states" to be rapidly decaying. In another related problem, an asymptotic derivation of the corresponding axisymmetric displacement boundary conditions for a certain class of orthotropic circular cylindrical shells (with vanishing transverse normal strain and therefore uniform transverse displacement across the shell thickness) can be found in [13].

In this paper, we describe a new method, completely different from those used in [9] and [12], for obtaining the appropriate boundary conditions for the interior solution for plate problems. By way of a reciprocity theorem in linear elasticity, we obtain a set of necessary conditions on the boundary data for the existence of a rapidly decaying solution of the plate problems. These necessary conditions are then translated into the desired set of boundary conditions for the interior expansion; they do not involve the edge zone corrections for the particular problem under consideration. In the case of displacement edge data, the boundary conditions for the interior solution do involve the solution of certain (properly formulated) canonical problems which can be solved once and for all. As such, our approach is similar in spirit to that of Kolos, though the actual methods of derivation are not at all related.

To describe the essential features of our method with a minimum amount of technical complications, we present in this paper only results for the special case of an infinite strip plate with boundary data uniform along its two straight edges. For this class of problems, our necessary conditions for a rapidly decaying solution are also asymptotically (as $\mathrm{E} \rightarrow 0$ ) sufficient conditions; therefore, the implied boundary condi- 
tions for the interior solution are asymptotically correct. ${ }^{2}$ For the stress boundary value problem, our boundary conditions along an edge coincide with those obtained in [9] and [12] for plate extension and bending, respectively. For the displacement boundary value problem, the appropriate edge conditions for the interior solution, not previously known in the literature, involve the solution of three canonical problems in two-dimensional linear elasticity, namely the stretching, bending and flexure of a semi-infinite strip with a built-in end. The solutions of these well-known problems have been worked out in the literature [14-18] (see also [19] for other references and some recent developments). Conditions on edge-data for a decaying state (and the implied edge conditions for the interior solution) are also obtained for two different sets of mixed edge-data. These results indicate how the appropriate boundary conditions for the interior solution might be derived for plates with curved edges and with edgewise nonuniform data. Some results for the more general case will be reported elsewhere.

In section (2), we obtain an eigenfunction expansion for the conventional class of plane strain states in a semi-infinite elastic strip in the absence of distributed or concentrated loads in the strip interior. This result is then used in section (3) to relate the non-decaying components of the plane strain states to the edge data. The necessary and sufficient conditions for a decaying state of plane strain given in section (4) follow from these relations.

To illustrate how these conditions for a decaying state may be used to obtain boundary conditions for the interior solution, we solve in section (5) a particular problem involving displacement edge-data. In this problem we consider the plane strain deformation of an infinite rectangular block whose sides suffer equal and opposite uniform displacements transverse to the upper and lower faces of the block. We calculate the interior solution (to within exponentially small error) and in particular we determine the resultant transverse force (per unit length) required at each edge to produce the prescribed edge displacement. Although our results are only asymptotically valid as $\boldsymbol{\varepsilon}$ (thickness/width for the block) tends to zero, the formula for the transverse force is less than $0.1 \%$ in error even when $\varepsilon=1$, the case of a square block!

In section (6), we describe the general interior expansion for an infinite strip plate in plane strain deformation, with its upper and lower faces free of tractions. Using the results of section (4), we derive the boundary conditions satisfied by the terms of this interior expansion for four different types of edge-data: stress data, displacement data, and two different sets of mixed data.

In section (7) we observe the remarkable fact that for the particular geometry ${ }^{3}$ of the infinite strip plate in plane strain deformation, the parametric expansion series for the interior solution can be summed to yield the governing equations and boundary conditions satisfied by the full interior solution on the mid-plane of the plate; these boundary conditions are derived not only for stress edge-data (where the classical St. Venant principle is proved to apply), but also for displacement and mixed edge-data. Thus for this geometry it is possible to proceed directly to the full interior solution (to

2 For a semi-infinite plate with edgewise uniform data along its only straight edge, our boundary conditions for the interior expansion are rigorously correct.

${ }^{3}$ We will show in a later communication that this simplification is also possible for the case of a circular plate with axisymmetric stress edgedata. 
within exponentially small error as $\varepsilon \rightarrow 0$ ), without determining any of the terms of the parametric series expansion.

Finally, to illustrate how our method is equally applicable to plates subject to prescribed surface loading, we solve in section (8) the problem of an infinite plate strip subject to uniform pressure on its faces, and with its edges rigidly fixed. We obtain the interior solution (to within exponentially small error) and in particular we find the deflection of the mid-plane of the plate. Even when $\varepsilon$ is quite small $(\varepsilon=1 / 5$ say), the discrepancy between the full interior solution and its leading term (the classical Kirchhoff plate theory) is as large as $40 \%$. Furthermore, the inclusion of the second term of the interior expansion (the $\mathrm{O}(\mathrm{E})$ correction term) serves only to increase this error unless $\varepsilon$ is exceptionally small. [Even when $\varepsilon=1 / \mathrm{SO}$, the classical Kirchhoff theory is more accurate than the $\mathrm{O}(\mathrm{E})$ corrected theory!] Although we have so far observed this effect only in the case of displacement edge-data, it does cast a doubt on the value of the $\mathrm{O}(\mathrm{E})$ corrected theory of Kolos for the other edge-data also ${ }^{4}$. We poined out earlier that it is difficult to go beyond $\mathrm{O}(\mathrm{E})$ correction terms with Kolos' method. In contrast, the approach described in this paper does not have the same difficulty.

\section{Regular state of plane strain in a semi-infinite strip}

Consider a semi-infinite strip of homogeneous, isotropic, linearly elastic material occupying the region $-\frac{1}{2} H \leqslant x_{3} \leqslant \frac{1}{2} H, x_{1} \geqslant 0$. The strip is in an equilibrium state of plane strain under no external load in the interior and no tractions along the long edges $x_{3}= \pm \frac{1}{2} H$. We introduce dimensionless variables $\mathrm{x}, \mathrm{z}$, defined by

$$
\begin{aligned}
& x_{1}=L x, \\
& x_{3}=\frac{1}{2} \mathrm{~Hz},
\end{aligned}
$$

where $L \gg H$ is some representative length in the xi-direction. The traction free conditions along the long edges of the strip then become

$$
\sigma_{x z}(x, \pm 1)=\sigma_{z z}(x, \pm 1)=0, \quad(x>0) .
$$

On the short end $x=0,|z|<1$, one of the following sets of conditions is prescribed:

$$
\begin{aligned}
& \text { Case (A): } \sigma_{x x}(0, z)=\bar{\sigma}_{x x}(z), \quad \sigma_{x z}(0, z)=\bar{\sigma}_{x z}(z), \quad(|z|<1), \\
& \operatorname{Case}(\mathrm{B}): \sigma_{x x}(0, z)=\bar{\sigma}_{x x}(z), u_{z}(0, z)=\bar{u}_{z}(z), \quad(|z|<1) \text {, } \\
& \text { Case }(C): u_{x}(0, z)=\bar{u}_{x}(z), \quad \sigma_{x z}(0, z)=\bar{\sigma}_{x z}(z), \quad(|z|<1) \text {, } \\
& \text { Case }(D): u_{x}(0, z)=\bar{u}_{x}(z), \quad u_{z}(0, z)=\bar{u}_{z}(z), \quad(|z|<1) \text {. }
\end{aligned}
$$

In this paper, we will be interested in those plane strain states which, in addition to satisfying the usual equilibrium and compatibility relations ${ }^{5}$ for plane strain and the

4 A similar phenomenon has been observed in the St. Venant edge zone expressions for the bounds of influence coefficients of an end-loaded cantilever beam [29] and of a semi-infinite circular cylindrical shell [30].

5 The usual compatibility relations require $\boldsymbol{\sigma}$ to have continuous second derivatives, but if we were to insist on this condition holding at the two corner points $(0, \pm 1)$, then there would in general be no solution corresponding to Case (D). In order not to exclude the actual solutions in this case we shall allow $\boldsymbol{\sigma}$ to have integrable (power law) singularities at $(0, \pm 1)$, while $\boldsymbol{u}$ remains continuous there (see [19], Theorem 1 ). 
above boundary conditions, ${ }^{6}$ also satisfy the

Growth Condition: As $x \rightarrow \infty$, the stress and displacement fields have at worst an algebraic growth.

We now define a class of plane strain states in terms of this growth condition:

Definition: An equilibrium state of plane strain $\{\boldsymbol{\sigma}, \boldsymbol{u}\}$ in the semi-infinite strip which satisfies the traction-free conditions (2.3) one choice of the prescribed end-data (2.4)-(2.7) and the growth condition (2.8) will be called a regular state of plane strain fitting the prescribed end-data.

It is important to observe that there are always infinitely many regular states of plane strain which fit any given set of (admissible) end-data. For example, the displacement field for Case (A) is determined up to a rigid body translation and rotation, a well known fact in the theory of elasticity. In Case (C), the end-data $u_{x}(0, \mathrm{z})=0, \sigma_{x z}(0, \mathrm{z})=0$ is fitted by $\left\{\boldsymbol{M \sigma} \boldsymbol{\sigma}^{V B}, \boldsymbol{M} \boldsymbol{u}^{V B}\right\}$ (where $\left\{\boldsymbol{\sigma}^{V B}, \boldsymbol{u}^{V B}\right\}$ is the St. Venant bending field given in the Appendix), for any value of the constant $M$. The reason for this is the growth condition (2.8), where we have allowed any algebraic growth in $\boldsymbol{\sigma}, \boldsymbol{u}$ as $\mathrm{x} \rightarrow \mathrm{cc}$. This is in sharp contrast to the usual kind of boundary value problem where a more restrictive condition than (2.8) would be imposed to ensure a unique solution. It follows that in the present paper we may not regard a particular choice of (admissible) end-data as "giving rise to" an associated regular state of plane strain in the semi-infinite strip since such a state is always non-unique. (The nature of this non-uniqueness will be described completely in section (3), Theorems (2A)-(2D).)

All regular states of plane strain in the semi-infinite strip may be characterized by the following expansion theorem which expresses $\boldsymbol{\sigma}$ and $\boldsymbol{u}$ in terms of the St. Venant fields for the unit tension, flexure and bending problems for an infinite strip with traction free edges (denoted by superscripts $V T, V F$ and $V B$, respectively), together with those Papkovich-Fadle eigenfunction fields of the same infinite strip which decay exponentially as $\mathrm{x} \rightarrow \mathrm{cc}$ :

Theorem (1): (i) For $x>0$, each regular state of plane strain $\{\boldsymbol{\sigma}, \boldsymbol{u}\}$ in the semi-infinite strip may be expressed in the form

$$
\begin{aligned}
\boldsymbol{\sigma} & \equiv\left(\begin{array}{ll}
\boldsymbol{\sigma}_{x x} & \sigma_{x z} \\
\boldsymbol{\sigma}_{z x} & \sigma_{z z}
\end{array}\right) \\
& =X \boldsymbol{\sigma}^{V T}+Z \boldsymbol{\sigma}^{V F}+M \boldsymbol{\sigma}^{V B}+\sum_{\lambda \in \mathscr{L}} A_{\lambda} \boldsymbol{\sigma}_{\lambda}+\sum_{\mu \in \mathscr{M}} B_{\mu} \boldsymbol{\sigma}_{\mu},
\end{aligned}
$$

\footnotetext{
$\overline{6}$ We shall always assume that the data functions $\overline{\boldsymbol{\sigma}}_{\boldsymbol{x} x}(z)$ etc. appearing in (2.4)-(2.7) are admissible data in the sense that there does exist some plane strain state in the semi-infinite strip which fits this data. We shall not be concerned here with the precise restrictions this requirement imposes upon the data functions. However it should be noted that these are merely smoothness restrictions; for example, the first author has proved in [20] that in Case (A) the data is admissible if the functions $\overline{\boldsymbol{\sigma}}_{x \boldsymbol{x}}(z), \overline{\boldsymbol{\sigma}}_{\boldsymbol{x} \boldsymbol{y}}(z)$ have second derivatives of bounded variation on $[-1,1]$.
} 


$$
\begin{aligned}
\boldsymbol{u} & \equiv\left[\begin{array}{l}
u_{x} \\
u_{z}
\end{array}\right] \\
& =X u^{V T}+Z u^{V F}+M u^{V B}+\left[\begin{array}{l}
U-\Theta z \\
W+\Theta \frac{x}{\varepsilon}
\end{array}\right]+\sum_{\lambda \in \mathscr{L}} A_{\lambda} u_{\lambda}+\sum_{\mu \in \mathscr{M}} B_{\mu} u_{\mu},
\end{aligned}
$$

where the coefficients $X, Z, M, U, W, \Theta,\left\{A_{\lambda}, \lambda \in \mathscr{L}\right\}$ and $\left\{B_{\mu}, \mu \in \mathscr{M}\right\}$ are uniquely determined and where $\left\{\boldsymbol{\sigma}_{\lambda}, \boldsymbol{u}_{\lambda}\right\}$ and $\left\{\boldsymbol{\sigma}_{\mu}, \boldsymbol{u}_{\mu}\right\}$ are the symmetric and antisymmetric Papkovich-Fadle eigenfunction fields which decay as $\mathrm{x} \rightarrow \infty$. (Both the St. Venant fields and the Papkovich-Fadle fields are given in the Appendix.)

(ii) In particular as $x \rightarrow \infty$, we have

$$
\begin{aligned}
& \mathrm{a} \sim X \boldsymbol{\sigma}^{V T}+Z \boldsymbol{\sigma}^{V F}+M \boldsymbol{\sigma}^{V B}+\mathrm{O}\left(\mathrm{e}^{-\beta x / \varepsilon}\right), \\
& \boldsymbol{u} \sim X \boldsymbol{u}^{V T}+Z \boldsymbol{u}^{V F}+M \boldsymbol{u}^{V B}+\left[\begin{array}{l}
U-\Theta z \\
W+\Theta \frac{x}{\varepsilon}
\end{array}\right]+\mathrm{O}\left(\mathrm{e}^{-\beta x / \varepsilon}\right),
\end{aligned}
$$

uniformly in $|z| \leqslant 1$, where $\varepsilon=H / 2 L$ and where $\beta(\simeq 2.106 .$. .) is the smallest positive real part of the roots of the equation $\sin (2 y)=(2 y)$.

[We note that $X, \mathrm{Z}$ and $M$ may be identified as the axial force, transverse force and bending moment (all per unit length in the $x_{2}$-direction) acting on any cross-section of the strip. The displacement field $(U-\Theta z, W+\Theta x / \varepsilon)^{\prime}$ is just a rigid body displacement with $U$, Wand $\Theta$ all having the dimension of length.]

Proof: To obtain the expansions (2.3) and (2.4), we recall that for any plane strain state, $\{\boldsymbol{\sigma}, \boldsymbol{u}\}$ is derivable from an Airy stress function $\chi$ (see [21] for the existence of $\chi$ ) satisfying

$$
\nabla^{2} \nabla^{2} \chi=0, \quad \nabla^{2}() \equiv \frac{4}{H^{2}}\left[\varepsilon^{2}()_{x x}+()_{, z=}\right]
$$

in the interior of the strip, since it is subject to no distributed or concentrated loads there. The traction free conditions at the upper and lower edge, (2.3), now take the form

$$
\frac{2}{H L} \chi,_{x z}=\frac{1}{L^{2}} \chi_{, x x}=0 \quad(x>0, z= \pm 1) .
$$

Since $\chi$ is nonunique to within a linear function $A x+B z+C$, it is possible, with no loss in generality, to find coefficients $\mathrm{X}, \mathrm{Z}$ and $M$ such that

$$
\chi=X \chi^{V T}+Z \chi^{V F}+M \chi^{V B}+\hat{\chi}
$$

where the St. Venant fields $\chi^{V T}$, etc., are given in the Appendix and $\hat{\chi}$ satisfies (2.13) and

$$
\hat{\chi}(x, \pm 1)=\hat{\chi},{ }_{z}(x, \pm 1)=0 \quad(x>0) .
$$

Evidently, $\hat{\chi}$ (like $\chi$ ) grows at most algebraically as $\mathrm{x} \rightarrow \infty$ uniformly in $|z| \leqslant 1$. It follows from an expansion theorem of [22] that $\hat{\chi}$ may be expressed in the form

$$
\hat{\chi}=\sum_{\lambda \in \mathscr{L}} A_{\lambda} \chi_{\lambda}(x, z)+\sum_{\mu \in \mathscr{M}} B_{\mu} \chi_{\mu}(x, z),
$$

where $\left\{\chi_{\lambda}, \lambda \in \mathscr{L}\right\}$ and $\left\{\chi_{\mu}, \mu \in \mathscr{M}\right\}$ are the Airy stress functions associated with the 
Papkovich-Fadle eigenfunctions for the infinite strip $|z| \leqslant 1$ with traction free sides. The sets $\mathscr{L}$ and $\mathscr{M}$ pick out those eigenfunctions which decay exponentially as x $\rightarrow$ cc (see also the Appendix). The required expansions (2.9), (2.10) now follow immediately from (2.15) (2.17).

To show that the coefficients in (2.9) and (2.10) are unique, we note that the terms of

$$
\begin{aligned}
u_{z}(x, 0)= & Z\left[-\frac{1-\nu}{8 G \varepsilon^{3}}\right] x^{3}+M\left[\frac{3-3 \nu}{8 G L \varepsilon^{3}}\right] x^{2}+\Theta \frac{x}{\varepsilon}+W \\
& +\sum_{\lambda \in \mathscr{L}} A_{\lambda} v_{\lambda}(x, 0)+\sum_{\mu \in \mathscr{M}} B_{\mu} v_{\mu}(x, 0)
\end{aligned}
$$

(where $\mathrm{G}$ is the shear modulus and $\mathrm{v}$ is Poisson's ratio) form an asymptotic scale as $x \rightarrow \infty$. The coefficients in the asymptotic series are unique since all the eigenvalues involved are (essentially) distinct. A similar argument applied to $u_{x}(x, 0)$ shows that $U$ and $X$ are also unique.

Finally, the asymptotic formulas (2.11) and (2.12) follow from the fact that $\hat{\chi}$ and all its derivatives are $\mathrm{O}\left(\mathrm{e}^{-\beta x / \varepsilon}\right)$ as $\mathrm{x} \rightarrow \infty$ uniformly in $|z| \leqslant 1$. This completes the proof of Theorem (1).

From the above characterization of a regular state of plane strain, we get immediately the following useful corollary which plays a role later in the boundary conditions for plate theory:

Corollary (1): (i) If $\boldsymbol{\sigma} \rightarrow 0$ as $\mathrm{x} \rightarrow \infty$, then

$$
\boldsymbol{\sigma}=\mathrm{O}\left(\mathrm{e}^{-\beta x / \varepsilon}\right), \quad \boldsymbol{u}=\left(\begin{array}{l}
U-\Theta z \\
W+\Theta \frac{x}{\varepsilon}
\end{array}\right)+\mathrm{O}\left(\mathrm{e}^{-\beta x / \varepsilon}\right)
$$

as $x \rightarrow$ cc uniformly in $|z| \leqslant 1$.

(ii) If $\boldsymbol{u} \rightarrow \mathbf{0}$ as $x \rightarrow \infty$, then

$$
\boldsymbol{\sigma}=\mathrm{O}\left(\mathrm{e}^{-\beta x / \varepsilon}\right), \quad \boldsymbol{u}=\mathrm{O}\left(\mathrm{e}^{-\beta x / \varepsilon}\right)
$$

as $\mathrm{x} \rightarrow \mathrm{cc}$ uniformly in $|z| \leqslant 1$.

It is not difficult to see that, instead of $\mathrm{x} \rightarrow \mathrm{co}$, we could have obtained the asymptotic results (2.19) and (2.20) with $\varepsilon \rightarrow 0$ for a fixed $x$. The latter is what we actually need and use later in sections (5)-(8).

While we do not make use of it in this paper, the next corollary of Theorem (1) gives an alternative characterization of the three St. Venant fields to the minimum energy characterization of Sternberg and Knowles [23].

Corollary (2): Let $\{\boldsymbol{\sigma}, \boldsymbol{u}\}$ be any state of plane strain in the infinite strip $(-1 \leqslant \mathrm{z} \leqslant$ $1,-\infty<x<\mathrm{cc}$ ) which is traction free on $\mathrm{z}= \pm 1$ and with no worse than an algebraic growth as $x \rightarrow \pm \infty$ uniformly for $|z| \leqslant 1$. Then

$$
\begin{aligned}
& \boldsymbol{\sigma}=X \boldsymbol{\sigma}^{V T}+Z \boldsymbol{\sigma}^{V F}+M \boldsymbol{\sigma}^{V B} \\
& \boldsymbol{u}=X \boldsymbol{u}^{V T}+Z \boldsymbol{u}^{V F}+M \boldsymbol{u}^{V B}+\left(\begin{array}{l}
U-\Theta z \\
W+\Theta \frac{x}{\varepsilon}
\end{array}\right) .
\end{aligned}
$$




\section{The dependence of the field as $x \rightarrow \infty$ on edge data}

In this section we relate the coefficients $X, \mathrm{Z}, M, U, W, \Theta$, associated with the non-decaying terms as $\mathrm{x} \rightarrow \infty$ (or as $\varepsilon \rightarrow 0$ for a fixed $\mathrm{x}$ ) in the expansions (2.9) (2.10) to the prescribed end-data on $\mathrm{x}=0$.

Theorem (2A): Any regular state of plane strain which fits the stress end-data $(A)$ must have

$$
\left.X=\frac{1}{2} H \int_{-1}^{1} \overline{\boldsymbol{\sigma}}_{x x} \mathrm{~d} z, \quad Z=\frac{1}{2} H \int_{-1}^{1} \overline{\boldsymbol{\sigma}}_{x z} \mathrm{~d} z, \quad M=-\frac{1}{4} H^{2}\right]_{-1}^{1} \overline{\boldsymbol{\sigma}}_{x x} z \mathrm{~d} z
$$

Furthermore any set of assigned values for $U, V, \Theta$ then determines this regular state of plane strain uniquely.

Theorem (2B): Any regular state of plane strain which fits the mixed end-data (B) must have

$$
\begin{aligned}
& X=\frac{1}{2} H \int_{-1}^{1} \bar{\sigma}_{x x} \mathrm{~d} z, \quad M=-\frac{1}{4} H^{2} \int_{-1}^{1} z \bar{\sigma}_{x x} \mathrm{~d} z \\
& W=\frac{3}{4} \int_{-1}^{1}\left[\left(1-z^{2}\right) \bar{u}_{z}+\frac{H}{12 G}\left\{(2-\nu) z^{3}-\frac{6}{5}(1-\nu) z\right\} \bar{\sigma}_{x x}\right] \mathrm{d} z .
\end{aligned}
$$

Furthermore any set of assigned values for $\mathrm{Z}, U, \Theta$ then determines this regular state of plane strain uniquely.

Theorem (2C): Any regular state of plane strain which fits the mixed end-data (C) must have

$$
\begin{aligned}
& Z=\frac{1}{2} H \int_{-1}^{1} \bar{\sigma}_{x z} \mathrm{~d} z \\
& U=\frac{1}{2} \int_{-1}^{1}\left[\frac{\nu}{4 G} z \bar{\sigma}_{x z}+\bar{u}_{x}\right] \mathrm{d} z, \\
& \Theta=-\frac{3}{2} \int_{-1}^{1}\left[z \bar{u}_{x}+\frac{H}{8 G}\left\{\nu z^{2}-\frac{2}{5}(4+\nu)\right\} \bar{\sigma}_{x z}\right] \mathrm{d} z .
\end{aligned}
$$

Furthermore any set of assigned values for $X, M, V$ then determines this regular state of plane strain uniquely.

It should be noted that in each of the Cases (A), (B), (C) the prescribed end-data determines uniquely a particular three of the six constants $X, Z, M, U, W, \Theta$, which characterize the behaviour of the strip at infinity; if the remaining three constants are given assigned values, then the prescribed end-data is fitted uniquely by a regular state of plane strain.

In Case (D), the situation is a little different. 
THEOREM (2D): Any regular state of plane strain which fits the displacement end-data (D) must have

$$
\begin{aligned}
& U=X U^{B T}+\frac{1}{2} H \int_{-1}^{1}\left[\sigma_{x x}^{B T}(0, z) \bar{u}_{x}+\sigma_{x z}^{B T}(0, z) \bar{u}_{z}\right] \mathrm{d} z, \\
& W=Z W^{B F}+\frac{2}{H} M W^{B B}+\frac{1}{2} H \int_{-1}^{1}\left[\sigma_{x x}^{B F}(0, z) \bar{u}_{x}+\sigma_{x z}^{B F}(0, z) \bar{u}_{z}\right] \mathrm{d} z, \\
& \Theta=\frac{1}{2} H Z \Theta^{B F}+M \Theta^{B B}+\frac{1}{4} H^{2} \int_{-1}^{1}\left[\sigma_{x x}^{B B}(0, z) \bar{u}_{x}+\sigma_{x z}^{B B}(0, z) \bar{u}_{z}\right] \mathrm{d} z,
\end{aligned}
$$

where $\left\{\boldsymbol{\sigma}^{B T}, \boldsymbol{u}^{B T}\right\}$, etc. are the unit tension, flexure, and bending fields of a semi-infinite strip with a built-in end $(\boldsymbol{u}(0, \mathrm{z})=\mathbf{0})$, and $U^{B T}, W^{B F}$, etc. are the constants $U, W$. $\Theta$ for these canonical problems. (These fields and constants are defined and discussed in the Appendix.) Furthermore, if $X, Z, M$ are given assigned values, then there is a unique regular state of plane strain fitting the end-data $(D)$; the remaining constants being determined from (3.4).

Proof: We shall first obtain in detail the third equation for Case (B) (the formula for $W$ ), the other formulae in Cases (A), (B), (C) being obtained in a similar (or easier) manner. All these formulae are obtained by applying the reciprocity theorem

$$
\sum_{i, j} \oint_{C_{\delta, A}}\left[u_{i}^{(1)} \sigma_{i j}^{(2)}-u_{i}^{(2)} \sigma_{i j}^{(1)}\right] n_{j} \mathrm{~d} s=0
$$

to the portion of the strip bounded by the closed contour $C_{\delta . A}$ shown in Fig. (1).

In (3.5) $\{\mathrm{n}$,$\} are the direction cosines of the outward normal to C_{\delta . A}$, and $\left\{\boldsymbol{\sigma}^{(k)}, \boldsymbol{u}^{(k)}\right\}(k=1,2)$ are any plane strain states sufficiently smooth inside the contour. For Case (B) we take $\left\{\boldsymbol{\sigma}^{(1)}, \boldsymbol{u}^{(1)}\right\}$ to be $\{\boldsymbol{\sigma}, \boldsymbol{u}\}$, any regular state of plane strain fitting

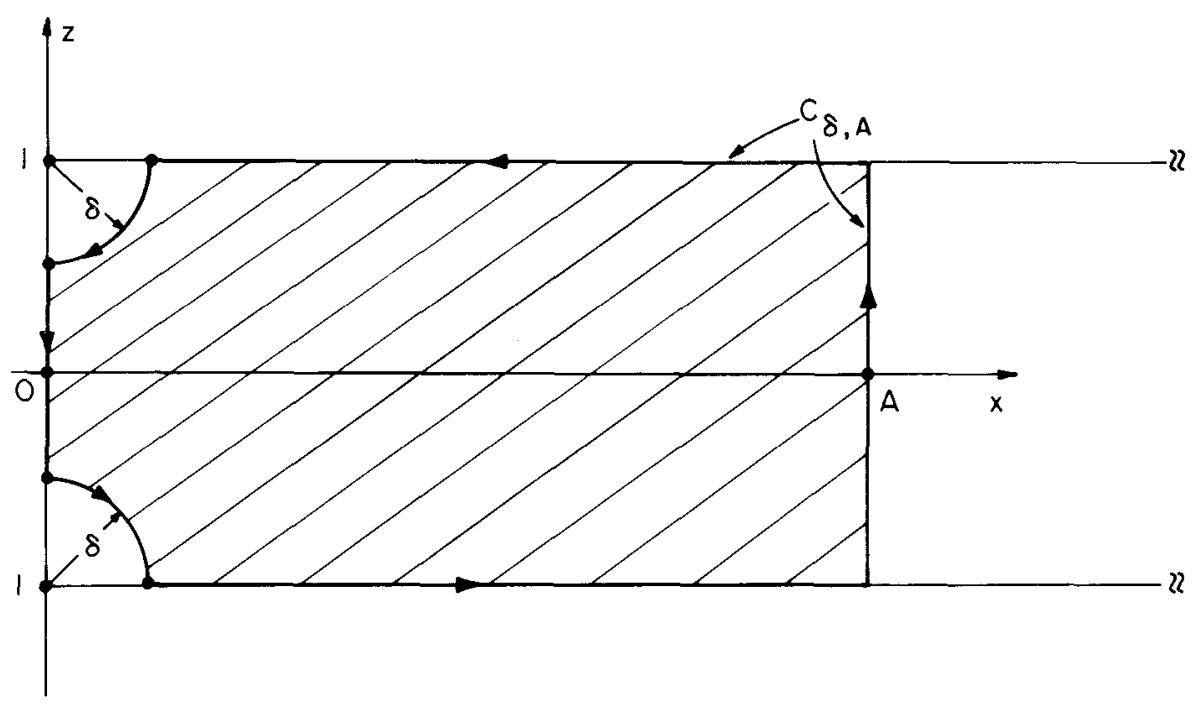

Figure 1. The contour of integration $C_{\delta . A}$. 
the prescribed end-data (2.5), and to obtain the formula for $W$ in (3.2c) we take $\left\{\boldsymbol{\sigma}^{(2)}, \boldsymbol{u}^{(2)}\right\}$ to be $\left\{\boldsymbol{\sigma}^{V I}, \boldsymbol{u}^{l ' F}\right\}$, the St. Venant field for unit flexure (given in the Appendix).

For these choices there is no contribution to the left side of (3.5) from the sides $\mathrm{z}= \pm 1, \delta<x<\mathrm{A}$ and there is no contribution from the quarter-circles near $(0, \pm 1)$ in the limit as $\delta \rightarrow 0$. What remains gives

$$
\begin{aligned}
& \int_{-1}^{1}\left[\bar{u}_{z} \sigma_{x z}^{V F}(0, z)-\bar{\sigma}_{x x} u_{x}^{V F}(0, z)\right] \mathrm{d} z \\
& \quad=\int_{-1}^{1}\left[u_{x} \sigma_{x x}^{V F}+u_{z} \sigma_{x z}^{V F}-u_{x}^{V F} \sigma_{x x}-u_{z}^{V F} \sigma_{x z}\right],{ }_{A} \mathrm{~d} z,
\end{aligned}
$$

since $\sigma_{x x}^{V F}(0, z)=u_{z}^{V F}(0, \mathrm{z})=0$.

In the right side of (3.6) we now replace $\{\boldsymbol{\sigma}, \boldsymbol{u}\}$ by the expansions (2.9), (2.10). The terms involving $X, Z, U, \Theta$ all give no contribution, and the remaining terms give

$$
\text { r.h.s. }=\frac{3(4+\nu)}{5 G H^{2}} M+\frac{2}{H} W+\mathrm{O}\left(A^{3} \mathrm{e}^{-\beta A}\right)
$$

as $\mathrm{A} \rightarrow$ cc. Letting $\mathrm{A} \rightarrow \infty$, we thus obtain

$$
W=\frac{1}{2} H \int_{-1}^{1}\left[\bar{u}_{z} \sigma_{x z}^{V F}(0, z)-\bar{\sigma}_{x x} u_{x}^{V F}(0, z)\right] \mathrm{dz}-\frac{3(4+\nu)}{10 G H} M .
$$

Upon taking the values of $\sigma_{x z}^{V F}(0, z), u_{x}^{V F}(0, z)$ from the Appendix. (3.8) will then yield the required formula (3.2c) once the formula (3.2b) for $M$ is established. This last formula can be deduced immediately from the conditions of overall equilibrium of the strip, or alternatively one may take $\left\{\boldsymbol{\sigma}^{(2)}, \boldsymbol{u}^{(2)}\right\}$ to be the rigid body displacement $\left\{\mathbf{0},\left(\begin{array}{r}-z \\ x\end{array}\right)\right\}$ in a much simpler version of the above argument. This establishes (3.2b).

(3.2c); (3.2a) could be obtained by taking $\left\{\boldsymbol{\sigma}^{(2)}, \boldsymbol{u}^{(2)}\right\}$ to be the rigid body displacement $\left\{0,\left(\begin{array}{l}1 \\ 0\end{array}\right)\right\}$

Finally, we observe that the three states $\left\{\boldsymbol{\sigma}^{V F}, \boldsymbol{u}^{V F}\right\} \cdot\left\{\mathbf{0},\left(\begin{array}{l}1 \\ 0\end{array}\right)\right\},\left\{\mathbf{0},\left(\begin{array}{r}-z \\ x\end{array}\right)\right\}$ each fit the end-data $\sigma_{x x}(0, z)=u_{z}(0, z)=0$ and so any linear combination of these states may be added to the original state $\{\boldsymbol{\sigma}, \boldsymbol{u}\}$ without disturbing the end-data (B) on $\mathrm{x}=0$. Hence it is possible to fit the end-data (B) and also take any assigned values of $\mathrm{Z}, U$, $\Theta$. When this is done, the resulting state is unique by the usual Kirchhoff energy argument (for a positive definite strain energy function).

Cases (A), (C) are proved in a similar manner.

In Case (D) we take $\left\{\boldsymbol{\sigma}^{(1)}, \boldsymbol{u}^{(1)}\right\}$ to be $\{\boldsymbol{\sigma}, \boldsymbol{u}\}$, any regular state of plane strain fitting the prescribed displacement end-data (2.7); $\left\{\boldsymbol{\sigma}^{(2)}, \boldsymbol{u}^{(2)}\right\}$ is taken to be successively $\left\{\boldsymbol{\sigma}^{B T}, \boldsymbol{u}^{B T}\right\},\left\{\boldsymbol{\sigma}^{B F}, \boldsymbol{u}^{B F}\right\},\left\{\boldsymbol{\sigma}^{B B}, \boldsymbol{u}^{B B}\right\}$, the unit tension, flexure and bending fields of a semi-infinite strip with a built-in end at $x=0$ (see Appendix). If we take the third choice we obtain

$$
\begin{aligned}
& \int_{-1}^{1}\left[\vec{u}_{x} \sigma_{x x}^{B B}(0, z)+\bar{u}_{z} \sigma_{x z}^{B B}(0, z)\right] \mathrm{d} z \\
& =\int_{-1}^{1}\left[u_{x} \sigma_{x x}^{B B}+u_{z} \sigma_{x z}^{B B}-u_{x}^{B B} \sigma_{x x}-u_{z}^{B B} \sigma_{x z}\right]_{x=A} \mathrm{~d} z .
\end{aligned}
$$


In the right side of (3.9) we replace $\left\{\boldsymbol{\sigma}^{B B}, \boldsymbol{u}^{B B}\right\}$ by the expansion (A3.2) to obtain

$$
\begin{aligned}
\text { r.h.s. }= & \int_{-1}^{1}\left[u_{x} \sigma_{x x}^{V B}+u_{z} \sigma_{x z}^{V B}-u_{x}^{V B} \sigma_{x x}-u_{z}^{V B} \sigma_{x z}\right]_{x=A} \mathrm{~d} z \\
& -\frac{2}{H} Z W^{B B}-\left(\frac{2}{H}\right)^{2} M \Theta^{B B}+O\left(A^{3} \mathrm{e}^{-\beta A}\right)
\end{aligned}
$$

as $A \rightarrow \infty$. In the integral in (3.10) we now replace $\{\boldsymbol{\sigma}, \boldsymbol{u}\}$ by the expansions (2.11), (2.12) to obtain

$$
\text { r.h.s. } \rightarrow\left(\frac{2}{H}\right)^{2} \Theta-Z\left\{\frac{2}{H} V^{B B}+\frac{3}{5 G H^{2}}(4+\nu)\right\}-\left(\frac{2}{H}\right)^{2} M \Theta^{B B},
$$

in the limit as $A \rightarrow \infty$. Combining (3.9), (3.11), we obtain

$$
\begin{aligned}
\left(\frac{2}{H}\right)^{2} \Theta= & Z\left\{\frac{2}{H} W^{B B}+\frac{3}{5 G H^{2}}(4+\nu)\right\}+\left(\frac{2}{H}\right)^{2} M \Theta^{B B} \\
& +\int_{-1}^{1}\left[\bar{u}_{x} \sigma_{x x}^{B B}(0, z)+\bar{u}_{z} \sigma_{x z}^{B B}(0, z)\right] \mathrm{d} z,
\end{aligned}
$$

which is not yet identical with (3.4c). Suppose however that we now apply (3.12) to the particular field $\left\{\boldsymbol{\sigma}^{B F}, \boldsymbol{u}^{B F}\right\}$ which fits the data $\bar{u}_{x}=\bar{u}_{z}=0$. Then $\Theta=\Theta^{B F}, \quad \mathrm{Z}=1$. $M=0$ and so

$$
\frac{4}{H^{2}} \Theta^{B F}=\frac{2}{H} W^{B B}+\frac{3}{5 G H^{2}}(4+\nu) .
$$

Substituting (3.13) into (3.12) then gives the required result $(3.4 \mathrm{c}) ;(3.4 \mathrm{a}, \mathrm{b})$ are obtained simularly.

Finally we observe that the three states $\left\{\boldsymbol{\sigma}^{B T \cdot B F \cdot B B}, \boldsymbol{u}^{B T \cdot B F . B B}\right\}$ each fit the end-data $\bar{u}_{x}=\bar{u}_{z}=0$ and so any linear combination of these states may be added to the original state $\{\boldsymbol{\sigma}, \boldsymbol{u}\}$ without disturbing the end-data $(D)$ on $\mathrm{x}=0$. Hence it is possible to fit the end-data $(D)$ and also take any assigned values of $X, Z, M$; when this is done, the resulting state is unique by the usual Kirchhoff energy argument.

Corollary (1): The constants $\Theta^{B F}, W^{B B}$ are related by

$$
\frac{2}{H} \Theta^{B F}-W^{B B}=\frac{3}{10 G H}(4+\nu) .
$$

This formula was obtained in the course of the preceding proof, Eqn. (3.13). We record it here for references elsewhere (see [19]).

Corollary (2): The constants $U^{B T}, W^{B F}, \Theta^{B F}, W^{B B}, \Theta^{B B}$ are related to their corresponding stress fields by

$$
\begin{aligned}
U^{B T} & =\frac{\nu H}{8 G} \int_{-1} z \sigma_{x z}^{B T}(0, z) \mathrm{d} z, \\
W^{B F} & =\frac{(2-\nu) H}{16 G} \int_{-1}^{1} z^{3} \sigma_{x x}^{B F}(0, z) \mathrm{d} z,
\end{aligned}
$$




$$
\begin{aligned}
& \Theta^{B F}=\frac{(2-\nu) H^{2}}{32 \mathrm{G}} \int_{-1}^{1} z^{3} \sigma_{x x}^{B B}(0, z) \mathrm{d} z+\frac{3}{4 G}, \\
& W^{B B}=-\frac{3 \nu}{8 G} \int_{-1}^{1} z^{2} \sigma_{x z}^{B F}(0, z) \mathrm{d} z, \\
& \Theta^{B B}=-\frac{3 \nu H}{16 G} \int_{-1}^{1} z^{2} \sigma_{x z}^{B B}(0, \mathrm{z}) \mathrm{d} \mathrm{z} .
\end{aligned}
$$

To prove (3.15), we write

$$
\left\{\boldsymbol{\sigma}^{B T}, \boldsymbol{u}^{B T}\right\}=\left\{\boldsymbol{\sigma}^{V T}, \boldsymbol{u}^{V T}\right\}+\left\{\boldsymbol{\sigma}^{* T}, \boldsymbol{u}^{* T}\right\}
$$

and apply the first condition of (3.4) to the "residual state" $\left\{\boldsymbol{\sigma}^{* T}, \boldsymbol{u}^{* T}\right\}$ which fits the data $\bar{u}_{x}=0$ and $\bar{u}_{z}=\nu z / 4 G$ and for which $\mathrm{X}=\mathrm{Z}=M=0$. The condition (3.15) follows immediately since $\left\{\boldsymbol{\sigma}^{B T}, \boldsymbol{u}^{B T}\right\}$ and $\left\{\boldsymbol{\sigma}^{* T}, \boldsymbol{u}^{* T}\right\}$ have the same value of $U$, namely $U^{B T}$. The relations (3.16)-(3.19) can be proved similarly.

It should be noted that the relations (3.15)-(3.19) are useful mainly for theoretical investigations. For the actual solution of the various clamped end problems, the rigid body displacements and rotation and the rest of the displacement and stress fields are to be determined simultaneously.

The formulae (3.1) which relate the growth behaviour of the plane strain state as $\mathrm{x} \rightarrow \mathrm{cc}$ to the resultant force and moment exerted at the short end by prescribed tractions, are a precise form of the St. Venant's principle (as applied to the semi-infinite strip); these formulae may alternatively be obtained by just considering the overall equilibrium of the strip. In the Cases (B), (C), (D) however, those formulae involving displacements at infinity (rather than resultant forces), namely (3.2c), (3.3b, c), (3.4a, b, c), cannot be derived by simple equilibrium considerations alone. Because of this the conventional form of St. Venant's principle is not directly useful for constructing the appropriate boundary conditions for plate theory in these cases, and previous limited attempts to derive such conditions have not been successful.

\section{Decaying states of plane strain in a semi-infinite strip}

Definition: A regular state of plane strain in the semi-infinite strip is said to be an (exponentially) decaying state if $\boldsymbol{u} \rightarrow 0$ as $\mathrm{x} \rightarrow \mathrm{cc}$ (which in turn implies $\boldsymbol{\sigma} \rightarrow 0$ ).

It follows from Corollary (1) of Theorem (1) that a regular state of plane strain is decaying if and only if $\mathrm{X}=\mathrm{Z}=M=U=W=\Theta=0$ so that, for a decaying state, $\{\boldsymbol{\sigma}, \boldsymbol{u}\}=\mathrm{O}\left(\mathrm{e}^{-\boldsymbol{\beta} x / \varepsilon}\right)$ as $\mathrm{x} \rightarrow$ cc uniformly in $-1 \leqslant z \leqslant 1$. (On the other hand, the condition $\boldsymbol{\sigma} \rightarrow 0$ alone does not guarantee a decaying state.) The following key result of this paper, with important implications in theory of plates and shells, is an immediate consequence of this observation: 
plane strain (in a semi-infinite strip with traction free sides) it is necessary to have

Case (A) : $\quad \frac{1}{2} H \int_{-1}^{1} \bar{\sigma}_{x x} \mathrm{~d} z=\frac{1}{2} H \int_{-1}^{1} \bar{\sigma}_{x z} \mathrm{~d} z=-\frac{1}{4} H^{2} \int_{-1}^{1} \overline{\boldsymbol{\sigma}}_{x x} z \mathrm{~d} z=0$.

Case (B) : $\quad \frac{1}{2} H \int_{-1}^{1} \bar{\sigma}_{x \cdot x} \mathrm{~d} z=-\frac{1}{4} H^{2} \int_{-1}^{1} \bar{\sigma}_{x x} z \mathrm{~d} z=0$,

$$
\int_{-1}^{1}\left[\frac{H}{12 G}(2-\nu) z^{3} \bar{\sigma}_{x, x}+\left(1-z^{2}\right) \bar{u}_{z}\right] \mathrm{d} z=0
$$

Case (C) : $\quad \frac{1}{2} H \int_{-1}^{1} \bar{\sigma}_{x z} \mathrm{~d} z=0$,

$$
\begin{aligned}
& \int_{-1}^{1}\left[\frac{\nu H}{4 G} z \bar{\sigma}_{x z}+\bar{u}_{x}\right] \mathrm{dz}=0, \\
& \int_{-1}^{1}\left[z \bar{u}_{x} \nmid \frac{n H_{x}}{8 G} z^{2} \bar{\alpha}_{z}\right] \mathrm{dz}=0,
\end{aligned}
$$

Case (D) : $\quad \frac{1}{2} H \int_{-1}^{1}\left[\sigma_{x x}^{B T}(0, z) \bar{u}_{x}+\sigma_{x z}^{B T}(0, z) \bar{u}_{z}\right] \mathrm{d} z=0$,

$$
\begin{aligned}
& \frac{1}{2} H \int_{-1}^{1}\left[\sigma_{x x}^{B F}(0, z) \bar{u}_{x}+\sigma_{x z}^{B F}(0, z) \bar{u}_{z}\right] \mathrm{d} z=0, \\
& \frac{1}{4} H^{2} \int_{-1}^{1}\left[\sigma_{x x}^{B B}(0, z) \bar{u}_{x}+\sigma_{x=}^{B B}(0, z) \bar{u}_{z}\right] \mathrm{d} z=0 .
\end{aligned}
$$

Furthermore, the prescribed end data surely induce a (unique) decaying state of plane strain in the semi-infinite strip if, in addition to the above necessary conditions, we have

Case (A): $\quad U=W=\Theta=0$

Case (B): $\quad Z=U=\Theta=0$

Case $(C): \quad X=M=W=0$

Case $(D)$ : One of $\{U, \mathrm{X}\}$ and two of $\{\mathrm{Z}, M, W, \Theta\}$ vanish.

Special cases of the necessary conditions (4.3) (4.5) and (4.6) have been obtained by Gusein-Zade [24,25] via a complete (formal) solution of the mixed boundary value problems (B) and (C). Our method of derivation of these conditions avoids the necessity of finding the complete solution of any boundary value problem and therefore applies to the displacement boundary value problem (D) and other problems not tractable by previously known approaches.

Theorem (3) has many far reaching implications. In the rest of this section, we confine ourselves to two immediate consequences of the results for Case (D). Others will be discussed in later sections of this paper.

EXAMPLE (i): The set of displacement end-data $\left\{\bar{u}_{x}=z, \bar{u}_{z}=0\right\}$ cannot be fitted by a decaying state of plane strain.

This follows since

$$
\int_{-1}^{1}\left[\sigma_{x x}^{B B}(0, z) \bar{u}_{x}+\sigma_{x z}^{B B}(0, z) \bar{u}_{z}\right] \mathrm{d} z=\int_{-1}^{1} z \sigma_{x x}^{B B}(0, z) \mathrm{d} z=\left(\frac{2}{H}\right)^{2},
$$

so that the necessarv condition (4.9) for the existence of a decaving state is not satisfied. 
For the second. we will need (a part of) the following preliminary results:

ThEOREM (4): $U^{B T} \leqslant 0, W^{B F}<0$ and $\Theta^{B B} \leqslant 0$ with equality holding only if $\mathrm{v}=0$.

P Roof: From the corollary (2) of theorem (2D). we have

$$
\begin{aligned}
U^{B T} & =\frac{\nu H}{8 G} \int_{-1}^{1} z \sigma_{x z}^{B T}(0, z) \mathrm{d} z=-\frac{1}{2} H \int_{-1}^{1} u_{z}^{l T}(0, z) \sigma_{x z}^{B T}(0, z) \mathrm{d} z \\
& =\frac{1}{2} H \int_{-1}^{1}\left[u^{* T}(0, z) \sigma_{x x}^{* T}(0, z)+u_{z}^{* T}(0, z) \sigma_{x=}^{* T}(0, z)\right] \mathrm{d} z,
\end{aligned}
$$

where again

$$
\left\{\boldsymbol{\sigma}^{* T}, \boldsymbol{u}^{* T}\right\}=\left\{\boldsymbol{\sigma}^{B T}, \boldsymbol{u}^{B T}\right\}-\left\{\boldsymbol{\sigma}^{V T}, \boldsymbol{u}^{V T}\right\}
$$

Transformed into a double integral of $\left[\boldsymbol{u} \cdot\left(\boldsymbol{\sigma}_{x x}, \boldsymbol{\sigma}_{x z}\right)^{t}\right],{ }_{x}+\left[\boldsymbol{u} \cdot\left(\boldsymbol{\sigma}_{y z}, \boldsymbol{\sigma}_{z z}\right)^{t}\right]$, by Green's theorem, the last integral across the strip width is seen to be $-2 W^{*} T$ where $W^{* T}$ is the strain energy of the residual state (per unit length in $x_{2}$-direction). Thus. we get $U^{B T} \leqslant 0$. The other two inequalities are proved similarly.

Example (ii): The set of displacement end-data $\left\{\bar{u}_{1}=0, \bar{u}_{z}=z^{2}\right\}$ cannot be fitted by a decaying state of plane strain if $v \neq 0$.

This follows because the integral

$$
\int_{-1}^{1}\left[\sigma_{x x}^{B B}(0, z) \bar{u}_{x}+\sigma_{x x}^{B B}(0, z) \bar{u}_{z}\right] \mathrm{d} z=\int_{-1}^{1} z^{2} \sigma_{x z}^{B B}(0, z) \mathrm{d} z=-\frac{16 G \Theta^{B B}}{3 v H}
$$

by (3.19), and is therefore positive by Theorem (4). Thus the condition (4.9) is again not satisfied so that the end-data cannot be fitted by a decaying state of plane strain.

These observations on the two sets of displacement end-data above are important in that they make it impossible to implement Kolos' method [9] of constructing appropriate boundary conditions for the classical or refined plate theory whenever displacement end-data are involved. The method in [9] requires the solution of several canonical probIems with "auxiliary problems" (4) and (5) involving prescribed displacement end-data along $\mathrm{x}=0$ and requiring $\boldsymbol{u} \rightarrow 0$ as $x \rightarrow \infty$. For auxiliary problem (4) the prescribed end-data is $\left\{\mathrm{ux}, \bar{u}_{-}\right\}=\left\{0, z^{2}\right\}$. For auxiliary problem (5) the prescribed end-data is $\left\{\bar{u}_{x}, \bar{u}_{z}\right\}=\{\mathrm{z}, \mathrm{O}\}$. These are just the displacement end-data analyzed above; they can not be fitted by a decaying state of plane strain, i.e., it is not possible to fit these data by a regular state of plane strain with $\boldsymbol{u} \rightarrow \mathbf{0}$ as $x \rightarrow \infty$. Therefore, it is not possible to implement the program outlined in [9] to get appropriate boundary conditions for plate theories if displacement data are involved.

The necessary conditions of Theorem (3) which render Kolos' method infeasible may themselves be transformed into an appropriate set of boundary conditions for plate theories whether we have stress or displacement boundary data. Indeed, we will show in section (6) of this paper how the appropriate set of boundary conditions for each term of the interior (or outer asymptotic) expansion of the exact solution may be obtained very simply from the necessary conditions of Theorem (3) without any reference to the boundary layer (or inner asymptotic) expansion. Before doing that, we first discuss in the next section a more direct application of Theorem (3) from which we will gain some insight to the results for the displacement end-data case. 


\section{The shearing of a rectangular block}

Consider a homogeneous, isotropic, linearly elastic rectangular block occupying the region $-L \leqslant x_{1} \leqslant L,-\infty<x_{2}<\infty$, and $-H / 2 \leqslant x_{3} \leqslant H / 2$. The top and bottom faces $x_{3}= \pm H / 2$ are free of tractions and the side faces $x_{1}= \pm L$ are bonded to rigid walls. The block is now placed in a state of plane strain by displacing the walls at $x_{1}= \pm L$ a distance $\pm w_{0}$ in the $x_{3}$-direction (uniformly in the $x_{2}$-direction). What forces and moments must be applied to the walls to produce these end displacements and what is the deformed shape of the block?

For the elastostatic behaviour of the block, it is sufficient to consider a layer of the block in a state of plane strain since the behaviour of the block is uniform in the $x_{2}$-direction. By the representation theorem established by the first author (see Theorem (4) of [22]), the displacement field $u(r)$ induced in the rectangular layer by the end displacements may be taken in the form

$$
\boldsymbol{u}(\boldsymbol{r})=Z \boldsymbol{u}^{V F}(\boldsymbol{r})+\Theta(-z, x / \varepsilon)^{\prime}+\sum_{\mu \in \mathscr{M}} B_{\mu} \boldsymbol{u}_{\mu}(\boldsymbol{r})-\sum_{\mu \in \mathscr{M}} B_{\mu} \boldsymbol{u}_{-\mu}(\boldsymbol{r})
$$

with the coefficients $Z, \Theta$ and $\left\{B_{\mu}\right\}$ completely (and uniquely) determined by the displacement data on $\mathrm{x}= \pm 1$ (where as before $\mathrm{x}=x_{1} / L, z=2 x_{3} / H$ and $\mathrm{E}=H / 2 L$ ), so that

$$
\boldsymbol{u}( \pm 1, z)=\left(0, \pm w_{0}\right)^{t}, \quad|z| \leqslant 1
$$

(For the present problem, $\mathrm{X}=M=U=W=A_{\lambda}=0, \lambda \in \mathscr{L}$, since $\boldsymbol{u}$ is odd in both $\mathrm{x}$ and z.) The first series in (5.1) consisting of $\boldsymbol{u}_{u}(\boldsymbol{r})$ terms decays exponentially like $\exp [-\gamma(x+1) / \varepsilon], \mathrm{y}=3.75 . . .$, as $\mathrm{x}$ increases from -1 , while the second decays exponentially like $\exp [-\gamma(1-x) / \mathrm{E} \mathbf{a s} \mathrm{x}$ decreases from 1 . Therefore, their contributions to $\boldsymbol{u}$ are expected to be negligible away from the two ends $(|\boldsymbol{r}-( \pm 1, z)|>2 \varepsilon$ say). We will regard the expression

$$
\boldsymbol{u}^{(I)}=Z \boldsymbol{u}^{V F}(\boldsymbol{r})+\Theta(-z, \mathrm{x} / \mathrm{c}),
$$

as the interior solution and the two series as end corrections at $\mathrm{x}= \pm 1$, respectively. Because an accurate determination of the constants $\left\{B_{\mu}\right\}$ is both difficult and tedious, an engineering approach to the problem would seek a solution of the form (5.3) above. We now see from (5.1) that this somewhat arbitrary semi-inverse approach is an attempt to obtain the portion of the full solution which is significant in the interior and to omit the end-corrections. The approach anticipates that the approximate solution obtained in this way would be close to the full solution except near $x= \pm 1$. The main difficulty of the semi-inverse procedure lies in the determination of the unknown constants $Z$ and $\Theta$ in terms of the prescribed end-data along $x= \pm 1,|z| \leqslant 1$.

Had the end-data been prescribed tractions, $Z$ could be chosen to give the known resultant force (and moment) applied to these ends. ( $\Theta$ is not determined by such end-data but may be fixed by some restriction on the end displacement, e.g., $u_{:}( \pm 1,0)$ $=0$.) St. Venant's principle would then be invoked to give some assurance that such a procedure would lead to an accurate approximate solution when $\varepsilon \ll 1$. We see from (5.1) (or (3.1)) that the procedure does in fact give the correct value for $\mathrm{Z}$.

When displacements are prescribed at the two ends $\mathrm{x}= \pm 1,|z|<1$ as in the present problem, it is not so easy to apply the above semi-inverse procedure to obtain the 
(approximate) interior solution. The expression (5.3) can not be made to satisfy the displacement end conditions (5.2) for any choice of $Z$ and $\Theta$. Nor can $Z$ be equated to the resultant transverse force applied by the wall (as in the prescribed traction case) since the end tractions required to produce the given end displacements are not known; indeed not even their resultants are known until we have solved the problem. The usual engineering approach (see [26] and similar texts) determines $\mathrm{Z}$ and $\Theta$ approximately by applying some special displacement conditions to the interior solution (5.3) at $x= \pm 1$. Such conditions are by no means unique. The following two choices are suggested in [26]:

$$
u_{z}( \pm 1,0)= \pm w_{0}, \quad u_{z . x}( \pm 1,0)=0
$$

or

$$
u_{z}( \pm 1,0)= \pm w_{0}, \quad u_{x, z}( \pm 1,0)=0
$$

These two choices lead to differing answers for the deformed shape of the rectangular sheet, neither of which fully satisfies the end conditions (5.2). More importantly, there is not a proper theoretical foundation for the special conditions (5.4) and (5.5) or any other set used in the literature since, at $x= \pm 1$, the end-corrections are comparable in magnitude with the interior solution and can not be neglected. Local conditions at the end should really be applied to the full solution (5.1), not just the interior solution (5.3) which is not expected to provide an accurate description of the correct end behaviour. While we may hope that the solution obtained by way of (5.4) or (5.5) would turn out to be a good approximation of (and possibly asymptotic to) the actual solution for small $\varepsilon$ (except near $x= \pm 1$ ), there seems to be no a priori reason for this to be so.

In view of the above discussion, it is rather gratifying that the results obtained in section (4) allow us to deduce in a rational way the correct interior solution (up to exponentially small terms when $\mathrm{E} \ll 1$ ) without any reference to the end-corrections. Let

$$
\begin{aligned}
& \left(\bar{u}_{x}^{(-)}(z), \bar{u}_{z}^{(-)}(z)\right)^{\prime}=\boldsymbol{u}^{(l)}(-1, z)-\left(0,-w_{0}\right)^{\prime} \\
& \quad=\frac{Z}{8 G \varepsilon^{3}}\left(\begin{array}{l}
3 \varepsilon(1-\nu) z-\varepsilon^{3}(2-\nu) z^{3}+6 \varepsilon^{3} z \\
(1-\nu)+3 \nu \varepsilon^{2} z^{2}
\end{array}\right)+\frac{\Theta}{\varepsilon}\left(\begin{array}{l}
-\varepsilon z \\
-1
\end{array}\right)+\left(\begin{array}{l}
0 \\
w_{0}
\end{array}\right) .
\end{aligned}
$$

If we now regard $\left(\bar{u}_{x}^{(-)} \bar{u}_{z}^{(}\right)^{-)}$as the displacement data on the end $\mathrm{x}=-1$ of the semi-infinite strip, $x \geqslant-1,|z| \leqslant 1$, then we know from theorem (3) that this set of end-data can be fitted by an exponentially decaying state in this strip only if $Z$ and $\Theta$ are so chosen that $\bar{u}_{x}^{(-)}$and $\bar{u}_{z}^{(-)}$satisfy the conditions (4.7))(4.9). By symmetry, this choice of $Z$ and $\Theta$ will also give rise to the same effect at $x=1$. The condition (4.7) is satisfied for any $\mathrm{Z}$ and $\Theta$ because of the symmetry of $\left(\bar{u}_{*}^{(-)}, \bar{u}_{z}^{(-)}\right)^{t}$. The other two conditions require

$$
\begin{aligned}
& \frac{Z}{8 G \varepsilon^{3}}\left\{(1-\nu)+3 \nu \varepsilon^{2} t_{2}^{F}+(\nu-2) \varepsilon^{3} n_{3}^{F}\right\}-\frac{1}{\varepsilon} \Theta+w_{0}=0 \\
& \frac{Z}{8 G \varepsilon^{2}}\left\{-3(1-\nu)+3 \nu \varepsilon t_{2}^{B}+(\nu-2) \varepsilon^{2} n_{3}^{B}-6 \varepsilon^{2}\right\}+\Theta=0
\end{aligned}
$$


Table 1

$\begin{array}{llllllll}\nu & t_{1}^{T} & t_{2}^{F} & n_{3}^{F} & t_{\mathbf{4}}^{F} & t_{2}^{B} & n_{3}^{B} & t_{4}^{B} \\ 1 / 4 & -0.0897 & 0.25393 & -0.07600 & -0.1332 & 0.07948 & -0.62311 & 0.074 \\ 1 / 3 & -0.1286 & 0.25024 & -0.07764 & \text { PO.1320 } & 0.11361 & -0.63015 & 0.107 \\ 1 / 2 & -0.2308 & 0.23880 & -0.08601 & -0.1280 & 0.20351 & -0.63882 & 0.190\end{array}$

where

$$
\begin{array}{ll}
n_{m}^{Y}=\frac{1}{2} H \int_{-1}^{1} z^{m} \sigma_{x x}^{B Y}(0, z) \mathrm{d} z, & n_{m}^{B}=\frac{1}{4} H^{2} \int_{-1}^{1} z^{m} \sigma_{x x}^{B B}(0, z) \mathrm{d} z, \\
t_{m}^{Y}=\frac{1}{2} H \int_{-1}^{1} z^{m} \sigma_{x z}^{B Y}(0, z) \mathrm{d} z, & t_{m}^{B}=\frac{1}{4} H^{2} \int_{-1}^{1} z^{m} \sigma_{x z}^{B B}(0, z) \mathrm{d} z,
\end{array}
$$

with $Y=T, F$ and $m=1, \ldots, 4$. The fields $\boldsymbol{\sigma}^{B T}, \boldsymbol{\sigma}^{B F}$, and $\boldsymbol{\sigma}^{B B}$ are defined in the Appendix and have been obtained in [17], [18] and [19]; ${ }^{7}$ the integrals (5.9) and (5.10) were obtained in [19]. The following table of values of the coefficients $n_{m}^{Y}$ and $t_{m}^{Y}$, $Y=T, B, F$ and $m=1,2,3,4$ for some typical values of $\nu$ is taken from [19] and is included for later references. By (3.15), (3.16), (3.19) and Theorem (4), we have $t_{1}^{T}<0$, $n_{3}^{F}<0$ and $t_{2}^{B}>0$ for $0<\nu \leqslant \frac{1}{2}$.

It follows from (5.7) and (5.8) that

$$
\begin{aligned}
& Z=\frac{8 G \varepsilon^{3} w_{0}}{2(1-\nu)-3 \nu \varepsilon t_{2}^{B}+\left[6-3 \nu t_{2}^{\prime}+(2-\nu) n_{3}^{B}\right] \varepsilon^{2}+(2-\nu) \varepsilon^{3} n_{3}^{F}} \\
& \Theta=\varepsilon w_{0} \frac{3(1-\mathrm{v})-3 \nu \varepsilon t_{2}^{B}+\left[(2-\nu) n_{3}^{B}+6\right] \varepsilon^{2}}{2(1-\nu)-3 \nu \varepsilon t_{2}^{B}+\left[6-3 \nu t_{2}^{F}+(2-\nu) n_{3}^{B}\right] \varepsilon^{2}+(2-\nu) \varepsilon^{3} n_{3}^{F}}
\end{aligned}
$$

Note that the expressions (5.11) and (5.12) are not the exact values of $\mathrm{Z}$ and $\Theta$ appearing in (5.1). The reason is that the exponentially decaying state, which fits the data $\left(\bar{u}_{x}^{(-)}, \bar{u}_{z}^{(-)}\right)^{t}$ on $x=-1$, itself gives rise to small (of order $\mathrm{e}^{-2 \gamma / t}$ as $E \rightarrow 0$ ) displacements on $\mathrm{x}=1$. and these displacements can not in general be fitted by a decaying state in the semi-infinite strip $x \leqslant 1,|z| \leqslant 1$. However, the resulting relative errors in the formulas (5.11) and (5.12) for $\mathrm{Z}$ and $\Theta$ are merely $\mathrm{O}\left(\mathrm{e}^{-2 \gamma / \varepsilon}\right)$ as $\varepsilon \rightarrow 0$. We would thus expect these formulas to be accurate to less that $0.1 \%$ in relative errors even for $\varepsilon=1$, the case of a square layer!

To compare the value of $\mathrm{Z}$ given by (5.11) with the corresponding ad hoc results obtained by way of (5.4) and (5.5), denoted by $Z_{1}$ and $Z_{2}$ respectively, we write (5.11) as

$$
\frac{4 G \varepsilon^{3} w_{0}}{(1-\nu) Z}=1-\frac{3 \nu t_{2}^{B}}{2(1-\nu)} \varepsilon+\frac{6-3 \nu t_{2}^{F}+(2-\nu) n_{3}^{B}}{2(1-\mathrm{v})} \varepsilon^{2}+\frac{(2-\nu) n_{3}^{F}}{2(1-\nu)} \varepsilon^{3}
$$

${ }^{7}$ The flexure solution in [17] and the bending solution in [18] contain computational errors; when corrected. agreement is obtained with [19]. 
while we have from [25]

$$
\frac{4 G \varepsilon^{2} w_{0}}{(1-\nu) Z_{1}}=1, \quad \frac{4 G \varepsilon^{3} w_{0}}{(1-\nu) Z_{2}}=1+\frac{3}{1-\nu} \varepsilon^{2}
$$

We see from (5.13)-(5.15) that both $Z_{1}$ and $Z_{2}$ give the correct leading term asymptotic solution for $\varepsilon \ll 1$. However, the second term in $(5.15)$, namely $3 \varepsilon^{2} /(1-\nu)$ is not the next term in the asymptotic expansion of $4 G \varepsilon^{3} w_{0} /(1-\nu) Z$.

Figure 2 shows the variation of $1 / Z$ with $\varepsilon(0<\varepsilon<1)$ for $v=1 / 4,1 / 3,1 / 2$. Figure 3 compares $Z_{1} / Z, Z_{1} / Z_{2}$ as functions of $\varepsilon(0<\varepsilon<1)$ for $\mathrm{v}=1 / 2$ (other values of $\mathrm{v}$ produced smaller differences between $Z, Z_{1}, Z_{2}$ ). It is interesting to note that as $\varepsilon \rightarrow 0$ the "first order corrected formula" for $Z$ is

$$
\frac{4 G \varepsilon^{3} w_{0}}{(1-\nu) Z}=1-\frac{3 \nu t_{2}^{B}}{2(1-\nu)} \varepsilon
$$

This is the formula for $Z$ which would be given by including the first correction term of refined plate theory (see section 6). However we see that, although (5.16) is the correct formula for $1 / Z$ (to order $\varepsilon$ ) as $\varepsilon \rightarrow 0$, unless $\varepsilon$ is extremely small (5.16) is a worse approximation to $1 / Z$ than the leading order term $1 / Z_{1}$; indeed the negative slope of $Z_{1} / Z$ at $\varepsilon=0$ indicated by (5.16) is not even visible in Fig. 2 . We shall return to this point in section (8). On the other hand $1 / Z_{2}$ given by (5.15), although devoid of theoretical foundation as an asymptotic approximation, is nevertheless quite a reasonable approximation to $1 / \mathrm{Z}$ in the range shown.

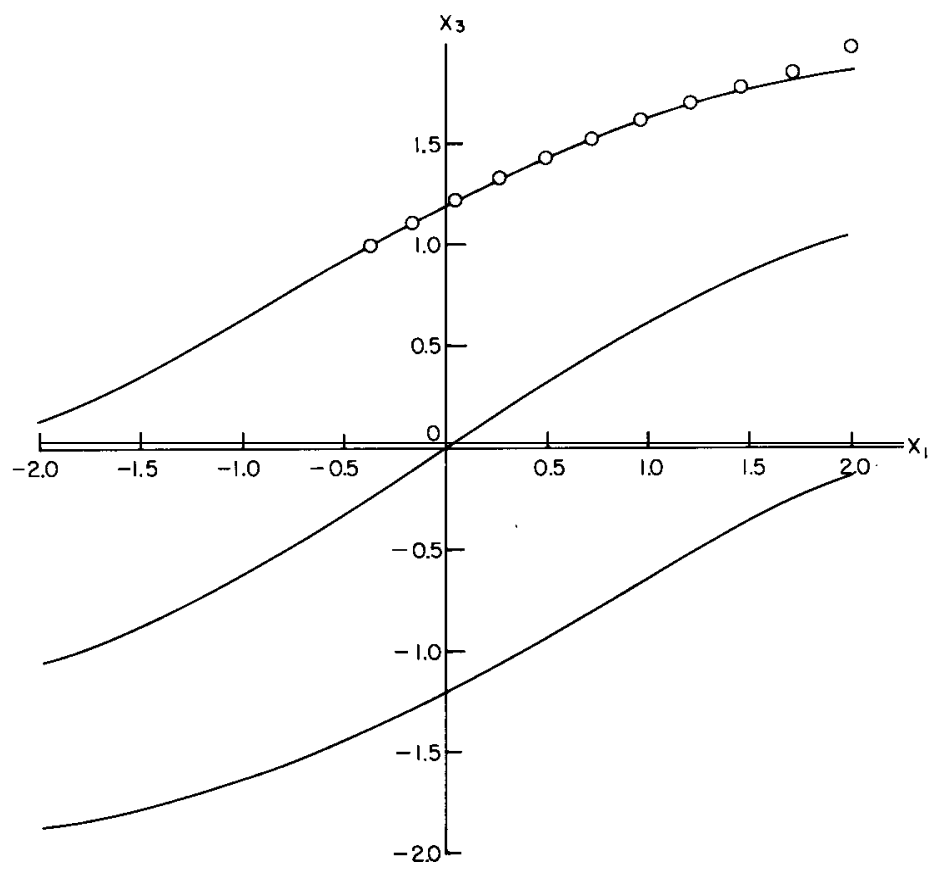

Figure 4. The deformed shape of the mid-plane, and upper and lower surfaces, of a block for which $\nu=1 / 2$, $H=2, L=2, w_{0}=1$, as calculated from the interior solution (5.3); the dots are the results of Dixit and Joseph [27]. 
The transverse displacement of the center line $\mathrm{z}=0$ calculated by using (5.11) and (5.12) for $\mathrm{Z}$ and $\Theta$ in (5.3) is

$$
u_{z}^{(I)}(x, 0)=w_{0} x \frac{\left(3-x^{2}\right)(1-\mathrm{v})-3 v t_{2}^{B} \varepsilon+\left[(2-\nu) n_{3}^{B}+6\right] \varepsilon^{2}}{2(1-v)-3 \nu t_{2}^{B} \varepsilon+\left[6-3 \nu t_{2}^{F}+(2-\nu) n_{3}^{B}\right] \varepsilon^{2}+(2-\nu) n_{3}^{F} \varepsilon^{3}},
$$

with the leading term of its expansion in powers of Ecorresponding to the result obtained by the ad hoc end conditions (5.4). In Fig. 4 we show the deformed shape of the mid-plane (and the upper and lower surfaces) of a block for which $\nu=1 / 2, L=2$, $H=2$ (so $\varepsilon=1 / 2$ ), and $w_{0}=1$, as calculated using (5.11), (5.12) for $\mathrm{Z}$ and $\Theta$. Here we are neglecting the "end effects" represented by the two series in (5.1); however from the discussion following (5.2) we expect these effects to be negligible for $\left|x_{1}\right|<1.5$. The dots in Fig. 4 represent the positions (after deformation) of the points of the upper surface of the block which, before deformation, had co-ordinates $x_{3}=1, x_{1}=0,0.2,0.4, \ldots, 1.8$, 2.0. These were calculated by Dixit and Joseph [27] as part of a full solution of the sheared block problem ${ }^{8}$ which included the end effects, and which involves the inversion of large matrices to compute the required solution. We see that, even when the aspect ratio $\varepsilon$ is as large as $1 / 2$, the agreement between our easily obtained interior solution and the full solution is excellent, and that the discrepancies are confined, as expected, to the narrow regions $1.5<\left|x_{1}\right|<2.0$.

We conclude this section by obtaining a more precise estimate for the errors in (5.13), (5.16). Upon substituting (5.11) and (5.12) into (5.6), we get

$$
\begin{aligned}
& \bar{u}_{x}^{( \pm)}(z)=\frac{1}{\Delta} \varepsilon^{2} w_{0} z\left[3 v t_{2}^{B}-(2-\nu) \varepsilon\left(z^{2}+n_{3}^{B}\right)\right], \\
& \bar{u}_{z}^{( \pm)}(z)= \pm \frac{1}{\Delta} \varepsilon^{2} w_{0}\left[3 v\left(t_{2}^{F}-z^{2}\right)-(2-v) \varepsilon n_{3}^{F}\right],
\end{aligned}
$$

with

$$
\mathrm{A}=2(1-\mathrm{v})-3 \nu \varepsilon t_{2}^{B}+\left[6-3 \nu t_{2}^{F}+(2-\nu) n_{3}^{B}\right] \varepsilon^{2}+(2-\nu) \varepsilon^{3} n_{3}^{F},
$$

as the boundary data for the (primary) residual stress states in the semi-infinite strips $\mathrm{x}<1$ and $\mathrm{x}>-1$, respectively. Since $\overline{\boldsymbol{u}}_{x}^{( \pm)}$and $\overline{\boldsymbol{u}}_{z}^{( \pm)}$satisfy (4.7)-(4.9), this data can be fitted in its respective semi-infinite strip by a decaying state, leaving a secondary residual displacement boundary value problem for the block with end-displacements of order $0\left(w_{0} \varepsilon^{2} \mathrm{e}^{-2 \gamma / \varepsilon}\right)$ as $\varepsilon \rightarrow 0$. Thus we expect the formulae (5.13), (5.16) to have relative errors of order

$$
O\left(\varepsilon^{2} \mathrm{e}^{-2 \gamma / \varepsilon}\right)
$$

as $\boldsymbol{E} \rightarrow 0$. When the aspect ratio $\varepsilon=1 / 2$ for instance, we have $\mathrm{e}^{-2 \gamma / t}=10^{-7}$, a remarkably small relative error!

\section{B oundary conditions for plate theory}

From a plate-theoretical viewpoint, the analysis of section (5), for a particular plane strain problem in a bounded domain, is significant in that it indicates how the results of 
section (4) may be used to derive the appropriate boundary conditions for the interior solution of boundary value problems for linearly elastic plates without any reference to the supplementary boundary layer solution. In this section, we derive these boundary conditions for a homogeneous, isotropic linearly elastic infinite plate strip occupying the region $-L \leqslant x_{1} \leqslant L,-\infty<x_{2}<\infty$, and $-H / 2 \leqslant x_{3} \leqslant H / 2$. The plate is free of tractions at its upper and lower faces, $\mathrm{x}_{3}= \pm H / 2$; the tractions and displacements along the two sides $x_{1}= \pm L$ are uniform in the $x_{2}$-direction and are such that they induce the same state of plane strain inside any rectangular layer cross-section, $\left|x_{1}\right|<L$. $\left|x_{3}\right|<H / 2$ (for any fixed value of $x_{2}$ ).

\section{Interior solution}

Consider generally a plate of uniform thickness $H$, free of external loads in the interior and free of surface tractions at its upper and lower faces $x_{3}= \pm H / 2$. It is well known from $[3,5,6,7]$ that the stress and displacement components of the interior (or outer asymptotic) expansion solution of the general boundary value problem in three dimensional linear elasticity theory for such a plate may be taken in the form

$$
\begin{aligned}
& \sigma_{i j}^{(I)}=\sigma_{0} \sum_{n=0}^{\infty} s_{i j}^{(n)} \varepsilon^{n}, \quad \sigma_{i z}^{(l)}=\sigma_{0} \sum_{n=0}^{\infty} s_{i z}^{(n)} \varepsilon^{n+1}, \quad \sigma_{z z}^{(I)}=\sigma_{0} \sum_{n=0}^{\infty} s_{z z}^{(n)} \varepsilon^{(n+2}, \\
& u_{i}^{(I)}=\frac{\sigma_{0} L}{E} \sum_{n=0}^{\infty} V_{i}^{(n)} \varepsilon^{n}, \quad u_{z}^{(I)}=\frac{\sigma_{0} L}{E} \sum_{n=0}^{\infty} W^{(n)} \varepsilon^{n-1}, \quad(i, j=x, y)
\end{aligned}
$$

where the coefficients of the parametric series are independent of $\varepsilon=H / 2 L$ and are functions of $\mathrm{x}=x_{1} / L, y=x_{2} / L$ and $z=x_{3} /(H / 2)$, and where $\sigma_{0}$ is a representative stress magnitude factor determined by the external loads. In general. the coefficients are given in terms of the mid-plane displacement quantities ${ }^{9} w^{(n)}(x, y), v_{x}^{(n)}(x, y)$ and $v_{v}^{(n)}(x, y)$ by

$$
\begin{aligned}
& W^{(n)}=\left\{w^{(n)}+\frac{\nu z^{2}}{2(1-\nu)} \nabla^{2} w^{(n-2)}\right\}-\frac{\nu z}{1-\nu}\left\{v_{x, x}^{(n-2)}+v_{y, y}^{(n-2)}\right\}, \\
& V_{x}^{(n)}=\left\{v_{x}^{(n)}+\frac{\nu z^{2}}{2(1-\nu)}\left[v_{x . x}^{(n-2)}+v_{1 . .}^{(n-2)}\right]_{. x}\right\} \\
& -\left\{z w_{. . .}^{(n)}+\frac{6 z+(\nu-2) z^{3}}{6(1-\nu)} \nabla^{2} w_{. . .}^{(n-2)}, \quad(x \leftrightarrow y)\right. \\
& s_{x, x}^{(n)}-\left\{n_{x, x}^{(n)}+\frac{\nu z^{2}}{2(1-\nu)} D_{x, v}\left[v_{x, x}^{(n-2)}+v_{y, y}^{(n-2)}\right]\right\} \\
& +\left\{z m_{x x}^{(n)}+\frac{6 z+(\nu-2) z^{3}}{6(1-v)} \nabla^{2} m_{x x}^{(n-2)}\right\}, \quad(x \leftrightarrow y)
\end{aligned}
$$

${ }^{9}$ For our purpose, it is more convenient to work with $v_{x}$ and $v_{y}$ instead of the Airy stress function as the primary unknowns for the portion of the solution which is associated with the in-plane extension action of the plate. 


$$
\begin{aligned}
s_{x y}^{(n)}= & \left\{n_{x, y}^{(n)}+\frac{\nu z^{2}}{2\left(1-\nu^{2}\right)}\left[v_{x, x}^{(n-2)}+v_{y, y}^{(n-2)}\right]_{x y}\right\} \\
& +\left\{z m_{x y}^{(n)}+\frac{6 z+(\nu-2) z^{3}}{6(1-\nu)} \nabla^{2} m_{x y}^{(n-2)}\right\}, \\
s_{x z}^{(n)}= & \frac{1}{2}\left(1-z^{2}\right) q_{x}^{(n)}, \quad(x \leftrightarrow y), s_{z z}^{(n)} \equiv 0 .
\end{aligned}
$$

where

$$
\begin{aligned}
& ()_{, t}=\frac{\partial()}{\partial t}, \quad \nabla^{2}()=()_{, x x}+()_{y y}, \\
& D_{x y}()=\frac{1}{1-\nu^{2}}\left[()_{x x}+\nu()_{. y y}\right], \\
& m_{x, x}^{(n)}=-D_{x, y} w^{(n)}, \quad(x \leftrightarrow y), \quad m_{x y}^{(n)}=-\frac{w_{x y}^{(n)}}{1+\nu}, \\
& q_{x}^{(n)}=-\frac{\nabla^{2} w_{, x}^{(n)}}{1-\nu^{2}}, \quad(x \leftrightarrow y), \\
& n_{x x}^{(n)}=\frac{v_{x, x}^{(n)}+\nu v_{y, y}^{(n)}}{1-\nu^{2}}, \quad(x \leftrightarrow y), \quad n_{x y}^{(n)}=\frac{v_{x, y}^{(n)}+v_{y, x}^{(n)}}{2(1+\nu)},
\end{aligned}
$$

with

$$
\begin{aligned}
& \nabla^{2} \nabla^{2} w^{(n)}=0, \\
& \nabla^{2} v_{x-}^{(n)}-\frac{1}{2}(1+\nu)\left(v_{x, y y}^{(n)}-v_{y, x x}^{(n)}\right)=0, \quad(x \leftrightarrow y) .
\end{aligned}
$$

In (6.2)-(6.13), quantities with a negative superscript vanish identically and the symbol $(\mathrm{x} \leftrightarrow \mathrm{y})$ indicates an equation similar to the preceding one with the role of $\mathrm{x}$ and $\mathrm{Y}$ interchanged.

Stress and displacement boundary conditions for the infinite plate strip

For the infinite plate strip in a state of plane strain uniform in the y-direction described earlier, it is appropriate to consider a layer cross-section of the infinite plate strip (for any fixed value of y). We consider a layer subject to the prescribed end tractions

$$
\sigma_{x x}(1, z)=\bar{\sigma}_{x x}^{(+)}(z), \quad \sigma_{x z}(1, z)=\bar{\sigma}_{x z}^{(+)}(z) \quad(|z|<1)
$$

at the end $x=1$, and subject at $x=-1$ to the prescribed end displacements

$$
u_{x}(-1, z)=\bar{u}_{x}^{(-)}(z), \quad u_{z}(-1, z)=\bar{u}_{z}^{(-)}(z) \quad(|z|<1) .
$$

In general, interior (outer) expansions of the exact solution of this boundary value problem, given by (6.1)-(6.13), can not be expected to satisfy the end conditions (6.14) and (6.15) exactly. Of interest in plate theory is an appropriate set of boundary conditions for the interior expansions so that the difference between the resulting interior solution and the exact solution of the problem (called the residual state henceforth) is significant only near the plate edges. Evidently, these boundary conditions should come from the necessary conditions for a decaying State of plane strain of section (4) and may be deduced from them by the procedure used in section (5). 
For the stress boundary conditions at $\mathrm{x}=1$, we regard as in section (5) the difference

$$
\left\{\boldsymbol{\sigma}_{x x}^{(+)}(z), \boldsymbol{\sigma}_{x z}^{(+)}(z)\right\} \equiv\left\{\boldsymbol{\sigma}_{x x}^{(I)}(1, z), \boldsymbol{\sigma}_{x z}^{(I)}(1, z)\right\}-\left\{\overline{\boldsymbol{\sigma}}_{x x}^{(+)}(z), \overline{\boldsymbol{\sigma}}_{x z}^{(+)}(z)\right\}
$$

as the stress end-data at $x=1$ of the semi-infinite strip $\mathrm{x} \leqslant 1,|z| \leqslant 1$. We know from Theorem (3) (case (A)) that this set of end-data can be fitted by an exponentially decaying state in the strip only if

$$
j_{-1}^{1} \boldsymbol{\sigma}_{x x}^{(+)}(\mathrm{z}) \mathrm{dz}=\int_{-1}^{1} \boldsymbol{\sigma}_{x z}^{(+)}(\mathrm{z}) \mathrm{dz}=\int_{-1}^{1} \boldsymbol{\sigma}_{x x}^{(+)}(z) z \mathrm{dz}=0 .
$$

The conditions (6.17) are effectively three boundary conditions for the interior solution and, with ()$_{, y} \equiv 0$, may be written as

$$
\begin{aligned}
& \left(\sigma_{0} H\right) \sum_{n=0}^{\infty}\left[n_{x x}^{(n)}+\frac{\nu}{6(1-\nu)} \nabla^{2} n_{x x}^{(n-2)}\right]_{x=1} \varepsilon^{n}=\int_{-H / 2}^{H / 2} \bar{\sigma}_{x x}^{(+)} \mathrm{d} x_{3} \equiv \bar{N}^{(+)}, \\
& \left(\frac{\sigma_{0} H^{2}}{6 L}\right) \sum_{n=0}^{\infty}\left[q_{x}^{(n)}\right]_{x=1}^{\varepsilon^{n}}=\int_{-H / 2}^{H / 2} \bar{\sigma}_{x z}^{(+)} \mathrm{d} x_{3} \equiv \bar{Q}^{(+)},
\end{aligned}
$$

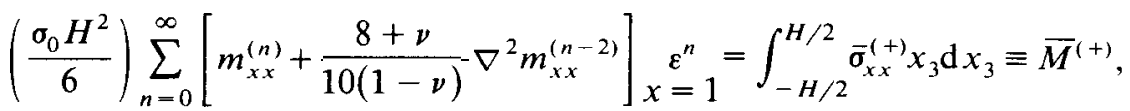

where $\nabla^{2}() \equiv() . x x$. The conditions (6.18)-(6.20) are merely the expected (and usually postulated) St. Venant principle (which requires the interior components to be equal to the prescribed end tractions in resultant forces and moments) for our plate strip but now as a direct consequence of the necessary conditions for the residual state to be a decaying state and significant only in a narrow region near the plate edge.

To treat the displacement boundary conditions at the other end $\mathrm{x}=-1$, we regard

$$
\left\{u_{x}^{(-)}(z), u_{z}^{(-)}(z)\right\} \equiv\left\{u_{x}^{(I)}(-1, z), u_{z}^{(I)}(-1, z)\right\}-\left\{\bar{u}_{x}^{(-)}(z), \bar{u}_{z}^{(-)}(z)\right\}
$$

as the displacement end-data at the end $x=-1$ of the semi-infinite strip $x \geqslant-1$ $|z| \leqslant 1$. We know from Theorem (3) (case (D)) that this set of end displacement dat can be fitted by an exponentially decaying state in the strip only if

$$
\begin{aligned}
& \int_{-1}^{1}\left[\sigma_{x x}^{B T}(0, z) u_{x}^{(-)}(z)+\sigma_{x z}^{B T}(0, z) u_{z}^{(-)}(z)\right] \mathrm{d} z=0, \\
& \int_{-1}^{1}\left[\sigma_{x x}^{B F}(0, z) u_{x}^{(-)}(z)+\sigma_{x z}^{B F}(0, z) u_{z}^{(-)}(z)\right] \mathrm{d} z=0, \\
& \int_{-1}^{1}\left[\sigma_{x x}^{B B}(0, z) u_{x}^{(-)}(z)+\sigma_{x z}^{B B}(0, z) u_{z}^{(-)}(z)\right] \mathrm{d} z=0 .
\end{aligned}
$$

Equations (6.21) are effectively three boundary conditions for the interior solution and with ()$_{, y} \equiv 0$, may be written as

$$
\begin{gathered}
\left(\frac{\sigma_{0} L}{E}\right) \sum_{n=0}^{\infty} \varepsilon^{n}\left\{v_{x}^{(n)}+\frac{\nu n_{2}^{T}}{2(1-\nu)} v_{x, x x}^{(n-2)}-\frac{\nu t_{1}^{T}}{1-\nu} v_{x, x}^{(n-1)}\right\}_{x=-1} \\
=\frac{1}{2} H \int_{-1}^{1}\left[\sigma_{x x}^{B T}(0, z) \bar{u}_{x}^{(-)}+\sigma_{x z}^{B T}(0, z) \bar{u}_{z}^{(-)}\right] \mathrm{dz} \equiv \bar{V}^{(-)},
\end{gathered}
$$




$$
\begin{aligned}
& \left(\frac{2 \sigma_{0} L^{2}}{E H}\right) \sum_{n=0}^{\infty} \varepsilon^{n}\left\{w^{(n)}+\frac{\nu t_{2}^{F}}{2(1-\nu)} \nabla^{2} w^{(n-2)}+\frac{(2-\nu) n_{3}^{F}}{6(1-\nu)} \nabla^{2} w_{. x}^{(n-3)}\right\}_{x=-1} \\
& =\frac{1}{2} H \int_{-1}^{1}\left\{\sigma_{x x}^{B F}(0, z) \bar{u}_{x}^{(-)}+\sigma_{x z}^{B F}(0, z) \bar{u}_{z}^{(-)}\right\} \mathrm{d} z \equiv \bar{W}^{(-)}, \\
& \frac{\sigma_{0} L}{E} \sum_{n=0}^{\infty} \varepsilon^{n}\left\{w_{\cdot x}^{(n)}+\frac{\nu t_{2}^{B}}{2(1-\nu)} \nabla^{2} w^{(n-1)}+\frac{6+(2-\nu) n_{3}^{B}}{6(1-\nu)} \nabla^{2} w_{. x}^{(n-2)}\right\}_{x=-1} \\
& =\frac{1}{4} H^{2} \int_{-1}^{1}\left\{\sigma_{x x}^{B B}(0, z) \bar{u}_{x}^{(-)}+\sigma_{x z}^{B B}(0, z) \bar{u}_{z}^{(-)}\right\} \mathrm{d} z \equiv \bar{\Omega}^{(-)},
\end{aligned}
$$

where the constants $n_{k}^{Y}$ and $t_{k}^{Y}$ are as defined in section (5).

The six relations, (6.18)-(6.20) and (6.22)-(6.24), decouple into two groups. Equations (6.18) and (6.22) involve only the quantities $n_{x x}^{(k)}$ and $v_{x}^{(k)}$ associated with the "in-plane stretching" plate action. For this group, we take $\boldsymbol{\sigma}_{0}$, the stress magnitude factor in (6.1), to be

$$
\sigma_{0}=\max \left\{\frac{\bar{N}^{(+)}}{H}, \frac{E \bar{V}^{(-)}}{L}\right\} \equiv \sigma_{S}
$$

and write

$$
\left\{\frac{\bar{N}^{(+)}}{\overline{H \sigma_{S}}}, \frac{E \bar{V}^{(-)}}{L \sigma_{S}}\right\} \equiv\left\{\bar{n}^{(+)}(\varepsilon), \vec{v}^{(-)}(\varepsilon)\right\}=\sum_{k=o}^{\infty}\left\{\bar{n}_{k}^{(+)}, \bar{v}_{k}^{(-)}\right\} \varepsilon^{k} .
$$

With (6.26), Eqns. (6.18) and (6.22) now give a set of two conditions for $v_{x}^{(k)}(x), k=0$, $1,2, \ldots$. In view of (6.13) and (6.11), which now read

$$
v_{x, x x}^{(k)}=0 \quad(-1<x<1), \quad \text { and } \quad n_{x x}^{(k)}=\frac{v_{x, x}^{(k)}}{1-\nu^{2}},
$$

respectively, these two boundary conditions reduce to

$$
\begin{gathered}
n_{x x}^{(k)}(1)=\frac{v_{x, x}^{(k)}(1)}{1-\nu^{2}}=\bar{n}_{k}^{(+)}, \\
\frac{\nu t_{1}^{T}}{}
\end{gathered}
$$

The second order differential equation for $v_{x}^{(k)}$ in (6.27) and the two boundary conditions (6.28) and (6.29) completely determine $v_{x}^{(k)}(x)$ and therewith $n_{x x}^{(k)}(x)$ (with $\left.n_{Y Y}^{(k)}=\nu n^{(k)}\right)$.

The r\&raining four equations, (6.19), (6.20), (6.23) and (6.24) involve only the quantities $w^{(k)}, m_{x x}^{(k)}$ and $q_{x}^{(k)}$ associated with the " transverse bending" plate action. For this group, we take

$$
\sigma_{0}=\max \left\{\frac{6 \bar{Q}^{(+)} L}{H^{2}}, \frac{6 \bar{M}^{(+)}}{H^{2}}, \frac{E H \bar{W}^{(-)}}{2 L^{2}}, \frac{E \bar{\Omega}^{(-)}}{L}\right\} \equiv \sigma_{B}
$$


and write

$$
\begin{aligned}
& \left\{\frac{6 \bar{Q}^{(+)} L}{H^{2} \sigma_{B}}, \frac{6 \bar{M}^{(+)}}{H^{2} \sigma_{B}}, \frac{E H \bar{W}^{(-)}}{2 L^{2} \sigma_{B}}, \frac{E \bar{\Omega}^{(-)}}{L \sigma_{B}}\right\} \equiv\left\{\bar{q}^{(+)}(\varepsilon), \bar{m}^{(+)}(\varepsilon), \bar{w}^{(-)}(\varepsilon), \bar{\omega}^{(-)}(\varepsilon)\right\} \\
& =\sum_{k=0}^{\infty}\left\{\bar{q}_{k}^{(+)}, \bar{m}_{k}^{(+)}, \bar{w}_{k}^{(-)}, \bar{\omega}_{k}^{(-)}\right\} \varepsilon^{k} .
\end{aligned}
$$

With (6.31), Eqns. (6.19), (6.20), (6.23) and (6.24) now give a set of four conditions for $w^{(k)}(x)$ for $k=0,1,2, \ldots$ In view of (6.12) (6.9) and (6.10) which are now simplified by ()$_{, y}=0$ to

$$
\begin{aligned}
& w_{. x x x \overline{\bar{x}}}^{(k)} \quad 0, \quad(-1<x<1) \\
& m_{x x}^{(k)}=-\frac{w_{. x x}^{(k)}}{1-\nu^{2}}, \quad q_{x}^{(k)}=-\frac{w_{. x x x}^{(k)}}{1-\nu^{2}},
\end{aligned}
$$

these four conditions become

$$
\begin{aligned}
& q_{x}^{(k)}(1)=-\frac{w_{, x x x}^{(k)}(1)}{1-\nu^{2}}=\bar{q}_{k}^{(+)}, \quad m_{x x}^{(k)}(1)=-\frac{w_{. x x}^{(k)}(1)}{1-\nu^{2}}=\bar{m}_{k}^{(+)}, \\
& w^{(k)}(-1)+\frac{\nu t_{2}^{F}}{2(1-\nu)} w_{, x x}^{(k-2)}(-1)+\frac{(2-\nu) n_{3}^{F}}{6(1-\nu)} w_{, x x x}^{(k-3)}(-1)=\bar{w}_{k}^{(-)}, \\
& w_{, x}^{(k)}(-1)+\frac{\nu t_{2}^{B}}{2(1-\nu)} w_{, x x}^{(k-1)}(-1)+\frac{6+(2-\nu) n_{3}^{B}}{6(1-\nu)} w_{, x x x}^{(k-2)}(-1)=\bar{\omega}_{k}^{(-)}
\end{aligned}
$$

The fourth order differential equation (6.32) for $w^{(k)}$ and the four boundary conditions (6.35)-(6.38) completely determine $w^{(k)}$ and therewith $m_{x x}^{(k)}$ and $q_{x}^{(k)}$ (with $m_{y y}^{(k)}=\nu m_{x x}^{(k)}$ and also (6.9)-(6.10)).

\section{Comparison with other results}

The stress boundary conditions (6.35) and (6.36) agree with those obtained in [9] ${ }^{10}$ and [12] when the latter results are specialized to the case of a plate with a straight edge and subject to edgewise uniform edge tractions (with $\overline{\boldsymbol{m}}_{k}^{(+)}=\bar{q}_{k}^{(+)}=0$ for $k \geqslant 1$ ). We note in particular that they do not involve higher or lower order terms of the various interior expansions. However, the unsimplified version of these same boundary conditions, namely (6.18)-(6.20), suggests that the stress boundary conditions for the kth order terms in these expansions may involve previously determined lower order terms when the edge tractions are edgewise non-uniform. This observation is at variance with the results in [12] but is supported by the $O(\boldsymbol{\varepsilon})$ correction terms for the classical plate theory obtained in [9]. The contributions of the lower order terms to the stress boundary conditions for the determination of successive coefficients of the interior expansions for plates with curved edge and more general loadings will be investigated by way of the reciprocity theorem in a separate publication.

Explicit results for various types of boundary conditions given by Kolos in [9] are only for the first two terms in the expansions (6.1). 
For $\mathrm{k}=0,1$ and $\overline{\boldsymbol{u}}_{x}^{(-)} \equiv \overline{\boldsymbol{u}}_{z}^{(-)} \equiv 0$, the displacement boundary conditions (6.37) and (6.38) agree with the form of the corresponding two term homogeneous conditions obtained by Kolos in [9] with $\boldsymbol{t}_{2}^{B}$ replacing Kolos' constant C. The method in [9] for determining $\mathrm{C}$ requires the fitting of a decaying state of plane strain to the displacement end data $\left\{\bar{u}_{x}=z, \bar{u}_{z}=0\right\}$ (auxiliary problem (5) in [9]) and another decaying state of plane strain to the end data $\left\{\bar{u}_{x}=0, \bar{u}_{z}=z^{2}\right\}$ (auxiliary problem (4) in [8]). We have already shown in section (4) that neither of these is possible. In contrast, the quantity $\boldsymbol{t}_{2}^{\boldsymbol{B}}$ is well-defined and has been computed in [19] (see also Table (1) in section (5) of this paper).

Having the inhomogeneous boundary conditions (6.22)-(6.24) for the entire interior solution, we now see that the displacement end data $\overline{\boldsymbol{u}}_{x}^{(-)}(z)$ and $\overline{\boldsymbol{u}}_{z}^{(-)}(z)$ contribute to both the in-plane stretching and transverse bending plate action. In contrast, the conventional approach simply matches the mid-plane displacements and rotation with their prescribed values so that

$$
\begin{aligned}
& v_{x}^{(0)}(-1)=\frac{E}{L \sigma_{S}} \bar{u}_{x}^{(-)}(0), \\
& w^{(0)}(-1)=\frac{E \varepsilon}{L \sigma_{B}} u_{z}^{(-)}(0), \quad w_{. x}^{(0)}(-1)=-\frac{E}{L \sigma_{B}} \bar{u}_{x, z}^{(-)}(0),
\end{aligned}
$$

completely neglecting possible contributions from $\Delta \bar{u}_{x} \equiv\left[\bar{u}_{x}^{(-)}(z)-\bar{u}_{x}^{(-)}(0)-z u_{x, z}^{(-)}(0)\right]$ and $\Delta \bar{u}_{z} \equiv\left[\bar{u}_{z}^{(-)}(z)-\bar{u}_{z}^{(-)}(0)\right]$. For example, the conventional displacement boundary conditions (6.39) give no interior stresses and displacements for the end data

$$
\begin{array}{ll}
\bar{u}_{x}^{(-)}(z)=U_{0}\left(z^{3}+z^{2}\right), & \bar{u}_{z}^{(-)}(z)=W_{0}\left(z^{2}+z^{3}\right), \\
\overline{\boldsymbol{\sigma}}_{x x}^{(+)} \equiv 0, & \overline{\boldsymbol{\sigma}}_{x z}^{(+)} \equiv 0,
\end{array}
$$

and effectively assume the corresponding state of plane strain is confined to an edge zone adjacent to the ends of the layer section. From (6.22)-(6.24), we have for this set of end data

$$
\begin{aligned}
& \bar{V}^{(-)}=U_{0} n_{2}^{T}+W_{0} t_{3}^{T}, \\
& \bar{W}^{(-)}=U_{0} n_{3}^{F}+W_{0} t_{2}^{F}, \\
& \bar{\Omega}^{(-)}=U_{0} n_{3}^{B}+W_{0} t_{2}^{B},
\end{aligned}
$$

which give rise to a nontrivial interior solution. The example suggests that the conventional displacement boundary conditions for plates (and shells) could lead to erroneous stress and displacement distributions even away from the plate edge(s). Moreover, we see from (6.41) that, in contrast to what we have been led to believe by the accepted results (6.39) of the conventional approach $\overline{\boldsymbol{u}}_{z}^{(-)}(z)$ would be primarily responsible for the in-plane stretching if $U_{0} \ll W_{0}$ !

Summary of boundary conditions for four types of edge-data

It should be noted that the stress magnitude factor $\boldsymbol{\sigma}_{0}$ in the interior expressions (6.1) is determined by the maximum of appropriate weighted averages of the prescribed data at the edges. For stress data $\left\{\overline{\boldsymbol{\sigma}}_{x x}^{(+)}(z), \overline{\boldsymbol{\sigma}}_{x z}^{(+)}(z)\right\}$ at $x=1$ and displacement data 
$\left\{\bar{u}_{x}^{(-)}(z), \bar{u}_{z}^{(-)}(z)\right\}$ at $x=-1$, we found $\sigma_{0}=\sigma_{S}$ for in-plane extension where $\sigma_{S}$ is given by

$$
\sigma_{S}=\max \left\{\frac{\bar{N}^{(+)}}{H}, \frac{E \bar{V}^{(-)}}{L}\right\},
$$

with

$$
\begin{aligned}
& \frac{2}{H} \bar{N}^{(+)}=\int_{-1}^{1} \bar{\sigma}_{x x}^{(+)} \mathrm{d} z, \\
& \frac{2}{H} \bar{V}^{(-)}=\int_{-1}^{1}\left[\sigma_{x x}^{B T}(0, z) \bar{u}_{x}^{(-)}+\sigma_{x z}^{B T}(0, z) \bar{u}_{z}^{(-)}\right] \mathrm{d} z .
\end{aligned}
$$

For transverse bending we found $\sigma_{0}=\sigma_{B}$, where

$$
\sigma_{B}=\max \left\{\frac{6 L \bar{Q}^{(+)}}{H}, \frac{6 \bar{M}^{(+)}}{H^{2}}, \frac{E H \bar{W}^{(-)}}{2 L^{2}}, \frac{E \bar{\Omega}^{(-)}}{L}\right\}
$$

with

$$
\begin{aligned}
& \left\{\frac{2}{H} \bar{Q}^{(+)}, \frac{4}{H^{2}} \bar{M}^{(+)}\right\}=\int_{-1}^{1}\left\{\bar{\sigma}_{x z}^{(+)}, z \bar{\sigma}_{x x}^{(+)}\right\} \mathrm{d} z, \\
& \left\{\begin{array}{c}
\frac{2}{H} \bar{W}^{(-)} \\
\frac{4}{H^{2}} \bar{\Omega}^{(-)}
\end{array}\right\}=\int_{-1}^{1}\left[\left\{\begin{array}{c}
\sigma_{x x}^{B F}(0, z) \\
\sigma_{x x}^{B B}(0, z)
\end{array}\right\} \bar{u}_{x}^{(-)}+\left\{\begin{array}{l}
\sigma_{x z}^{B F}(0, z) \\
\sigma_{x z}^{B B}(0, z)
\end{array}\right\} \bar{u}_{z}^{(-)}\right] \mathrm{d} z .
\end{aligned}
$$

(Recall from section (1) of the appendix that the dimensions of $\boldsymbol{\sigma}_{x x}^{B F}, \boldsymbol{\sigma}_{x z}^{B F}, \boldsymbol{\sigma}_{x x}^{B T}$ and $\boldsymbol{\sigma}_{x z} \boldsymbol{B T}$ are $1 /$ length and the dimensions of $\boldsymbol{\sigma}_{x x}^{B B}$ and $\boldsymbol{\sigma}_{x z}^{B B}$ are $1 /(\text { length })^{2}$.)

Once the stress magnitude factor $\boldsymbol{\sigma}_{\mathbf{0}}$ is determined for a particular set of prescribed data at the edges, the appropriate boundary conditions at $x= \pm 1$ for individual terms in the interior expansions (6.1) may be obtained by the procedure used above for the sample case with stress data at $x=1$ and displacement data at $x=-1$. We summarize below the appropriate edge conditions for these individual terms at $x=-1$ corresponding to the four types of edge data (2.4)-(2.7). (The same conditions with suitable sign changes apply to the $\mathrm{x}=1$ edge.)

\section{In - Plane Extension}

$$
v_{x, x x}^{(k)}=0, \quad|x|<1
$$

Case (A): Stress Data, $\overline{\boldsymbol{\sigma}}_{x x}$ and $\overline{\boldsymbol{\sigma}}_{x z}$ Prescribed

$$
x \subset-1: \quad n_{x x}^{(k)}=\frac{v_{-x, x}^{(k)}}{1-\nu^{2}}=\bar{n}_{k}, \quad(\mathrm{k}=0,1,2, \ldots),
$$

with (see also (6.43a))

$$
\frac{1}{2 \sigma_{S}} \int_{-1}^{1} \bar{\sigma}_{x x} \mathrm{~d} z \equiv \frac{\bar{N}}{H \sigma_{S}}=\sum_{k=0}^{\infty} \bar{n}_{k} \varepsilon^{k} .
$$


Case (B): Mixed Data, $\overline{\boldsymbol{\sigma}}_{x \boldsymbol{x}}$ and $\overline{\boldsymbol{u}}_{z}$ Prescribed

$$
x=-1: \quad n_{x x}^{(k)}=\frac{v_{-x, x}^{(k)}}{1-v^{2}}=\bar{n}_{k} \quad(k=0,1,2, \ldots) .
$$

Case (C): Mixed Data, $\overline{\boldsymbol{\sigma}}_{x z}$ and $\overline{\boldsymbol{u}}_{\boldsymbol{x}}$ Prescribed

$$
x=-1: \quad v_{x}^{(k)}=\bar{u}_{k}+\nu(1+\nu) \bar{t}_{k} \quad(k=O, 1,2, \ldots),
$$

with

$$
\begin{aligned}
& \frac{E}{2 L \sigma_{S}} \int_{-1}^{1} \bar{u}_{x} \mathrm{~d} z \equiv \frac{E \bar{U}}{L \sigma_{S}}=\sum_{k=0}^{\infty} \bar{u}_{k} \varepsilon^{k}, \\
& \frac{H}{4 L \sigma_{S}} \int_{-1}^{1} z \bar{\sigma}_{x z} \mathrm{~d} z \equiv \frac{\bar{T}}{\sigma_{S} L H}=\sum_{k=0}^{\infty} \bar{t}_{k} \varepsilon^{k} .
\end{aligned}
$$

(Thus the contribution of $\overline{\boldsymbol{\sigma}}_{\boldsymbol{x} z}$ to in-plane extension may predominate over that of $\overline{\boldsymbol{u}}_{x}$ in this case.)

Case (D): Displacement Data, $\overline{\boldsymbol{u}}_{\boldsymbol{x}}$ and $\overline{\boldsymbol{u}}_{z}$ Prescribed

$$
x=-1: \quad v_{x}^{(k)}-\frac{\nu t_{1}^{T}}{1-\nu} v_{x, x}^{(k-1)}=\bar{v}_{k} \quad(k=0,1,2, \ldots),
$$

with (see also (6.43b))

$$
\frac{E \bar{V}}{L \sigma_{S}}=\sum_{k=0}^{\infty} \bar{v}_{k} \varepsilon^{k}
$$

(Thus the contribution of $\overline{\boldsymbol{u}}_{z}$ to in-plane extension may predominate over that of $\overline{\boldsymbol{u}}_{x}$ in this case.)

\section{Transverse BENDING}

$$
w_{. x x x \bar{x}}^{(k)} \quad 0, \quad|x|<1
$$

Case (A): Stress Data, $\overline{\boldsymbol{\sigma}}_{\boldsymbol{x} \boldsymbol{x}}$ and $\overline{\boldsymbol{\sigma}}_{\boldsymbol{x} z}$ Prescribed

$$
\begin{gathered}
x=-1: \quad m_{x x}^{(k)}=-\frac{w_{, x x}^{(k)}}{1-\nu^{2}}=\bar{m}_{k}, q_{x}^{(k)}=-\frac{w_{1}^{(k)} x_{x x}}{1-\nu^{2}}=\bar{q}_{k} \\
(k=0,1,2, \ldots),
\end{gathered}
$$

with (see also (6.45a))

$$
\left\{\frac{6 L \bar{Q}}{H^{2} \sigma_{B}}, \frac{6 \bar{M}}{H^{2} \sigma_{B}}\right\}=\sum_{k=0}^{\infty}\left\{\bar{q}_{k}, \bar{m}_{k}\right\} \varepsilon^{k} .
$$

Case (B): Mixed Data, $\overline{\boldsymbol{\sigma}}_{\boldsymbol{x} \boldsymbol{x}}$ and $\overline{\boldsymbol{u}}_{\mathbf{2}}$ Prescribed

$$
m_{x x}^{(k)}=-\frac{w_{, x x}^{(k)}}{1-\nu^{2}}=\bar{m}_{k}
$$

$x=-1:$

$$
w^{(k)}=\bar{w}_{k}^{(2)}+(2-\nu)(1+\nu) \bar{m}_{k}^{(3)}-\frac{1-\nu^{2}}{5} \bar{m}_{k-2},
$$

$(\mathrm{k}=0,1,2, \ldots)$ 
with (see also (6.45a))

$$
\begin{aligned}
& \frac{3 E H}{8 L^{2} \sigma_{B}} \int_{-1}^{1}\left(1-z^{2}\right) \bar{u}_{z} \mathrm{~d} z \equiv \frac{3 E H \bar{W}^{(2)}}{8 L^{2} \sigma_{B}}=\sum_{k=0}^{\infty} \bar{w}_{k}^{(2)} \varepsilon^{k}, \\
& \frac{H^{2}}{16 L^{2} \sigma_{B}} \int_{-1}^{1} z^{3} \bar{\sigma}_{x x} \mathrm{~d} z \equiv \frac{\bar{M}^{(3)}}{4 L^{2} \sigma_{B}}=\sum_{k=0}^{\infty} \bar{m}_{k}^{(3)} \varepsilon^{k}
\end{aligned}
$$

Case (C): Mixed Data, $\overline{\boldsymbol{\sigma}}_{x z}$ and $\overline{\boldsymbol{u}}_{x}$ Prescribed $x=-1$ :

$$
\begin{aligned}
& q_{x}^{(k)}=-\frac{w_{, x x x}^{(k)}}{1-\nu^{2}}=\bar{q}_{k}, \quad(k=0,1,2, \ldots) \\
& w_{. x}^{(k)}=-\bar{u}_{k}^{(1)}-\nu(1+\nu) \bar{t}_{k}^{(2)}+\frac{1}{5}(4+\nu)(1+\nu) \bar{q}_{k-2}, \\
& \quad(k=0,1,2, \ldots)
\end{aligned}
$$

with (see also (6.45a))

$$
\begin{aligned}
& \frac{3 E}{2 \sigma_{B} L} \int_{-1}^{1} z \bar{u}_{x} \mathrm{~d} z \equiv \frac{E \bar{U}^{(1)}}{\sigma_{B} L}-\sum_{k=0}^{\infty} \bar{u}_{k}^{(1)} \varepsilon^{k}, \\
& \frac{3 H}{8 L \sigma_{B}} \int_{-1}^{1} z^{2} \bar{\sigma}_{x z} \mathrm{~d} z \equiv \frac{3 \bar{T}^{(2)}}{2 \sigma_{B} L H}=\sum_{k=0}^{\infty} \bar{t}_{k}^{(2)} \varepsilon^{k} .
\end{aligned}
$$

Case (D): Displacement Data. $\bar{u}_{-\bar{x}}$ and $i_{-\varsigma}$ Prescribed $\mathrm{x}--1$ :

$$
\begin{aligned}
& w^{(k)}+\frac{\nu t_{2}^{F}}{2(1-\nu)} w_{. x x}^{(k-2)}+\frac{(2-\nu) n_{3}^{F}}{6(1-\nu)} w_{. x x x}^{(k-3)}=\bar{w}_{k}, \quad(k=0,1,2, \mid .0) \\
& w_{. x}^{(k)}+\frac{\nu t_{2}^{B}}{2(1-\nu)} w_{, x x}^{(k-1)}+\frac{6+(2-\nu) n_{3}^{B}}{6(1-\nu)} w_{. x x x}^{(k-2)}=\bar{\omega}_{k}, \\
& (k=O, 1,2, \ldots)
\end{aligned}
$$

where (see also $(6.45 \mathrm{~b}))$

$$
\left\{\frac{E H \bar{W}}{2 L^{2} \sigma_{B}}, \frac{E \bar{\Omega}}{L \sigma_{B}}\right\}=\sum_{k=0}^{\infty}\left\{\bar{w}_{k}, \bar{\omega}_{k}\right\} \varepsilon^{k} .
$$

\section{Boundary conditions for the mid-plane interior solution}

In section (6), we have obtained the boundary conditions which must be applied to the individual terms of the parametric expansion (6.1) of the interior solution at a (straight) edge of an infinite strip plate in order for the residual state to be a rapidly decaying state. The plate strip considered there is free of external loads in the interior and free of surface tractions at its upper and lower faces; the two long edges of the plate strip are subject to edgewise uniform tractions and/or displacements so that the plate is in a state of plane strain. It is a remarkable fact that, in this two-dimensional situation, the 
parametric expansion can be summed to yield the boundary conditions which must be applied to the full mid-plane interior solution to ensure a decaying residual state; the boundary layer correction, although comparable with the interior solution near the edge, again need not be considered.

In this section, we summarize the appropriate boundary conditions for the mid-plane interior solution for the four types of end-data considered throughout this paper. In order for the results to be immediately useful in actual applications, we will now revert back to the unscaled co-ordinates $x_{1}, x_{3}$ (rather than the dimensionless co-ordinates $\mathrm{x}, \mathrm{z}$ defined by (2.1), (2.2)), and we will express our boundary conditions in terms of the components of the physical displacement $\boldsymbol{u}\left(x_{1}, x_{3}\right)$ (rather than the dimensionless displacements in (6.1)).

We shall again suppose that the plate occupies the region $-L \leqslant x_{1} \leqslant L,-\infty<x_{2}<$ $\infty,-\frac{1}{2} H \leqslant x_{3} \leqslant \frac{1}{2} H$, with the faces $x_{3}= \pm \frac{1}{2} H$ traction free. We consider the end $x_{1}=-L$, where we impose one of the four sets of boundary conditions (A), (B), (C), (D); similar conditions with suitable sign changes apply at the $x_{1}=\mathrm{L}$ end.

\section{In - Plane Extension}

The governing equation for in-plane extension may be taken to be

$$
\tilde{u}_{x .11}=0
$$

where $\tilde{u}_{x}\left(x_{1}\right)$ is the mid-plane displacement $u_{x}\left(x_{1}, 0\right)$. At $x_{1}=-L$, the appropriate boundary condition for plate extension is given below for four canonical sets of boundary data in 3-dimensional elasticity theory:

Case (A): Stress-Data, $\sigma_{x x}\left(-L, x_{3}\right)=\bar{\sigma}_{x x}\left(x_{3}\right), \sigma_{x z}\left(-L, x_{3}\right)=\bar{\sigma}_{x z}\left(x_{3}\right)$

$$
\tilde{u}_{x, 1}(-L)=\frac{1-\nu^{2}}{E H} \int_{-H / 2}^{H / 2} \bar{\sigma}_{x x} \mathrm{~d} x_{3} \text {. }
$$

Case (B): Mixed Data, $\sigma_{x x}\left(-L, x_{3}\right)=\bar{\sigma}_{x x}\left(x_{3}\right), u_{z}\left(-L, x_{3}\right)=\bar{u}_{z}\left(x_{3}\right)$

$$
\tilde{u}_{x, 1}(-L)=\frac{1-\nu^{2}}{E H} \int_{-H / 2}^{H / 2} \overline{\boldsymbol{\sigma}}_{x x} \mathrm{~d} x_{3} \text {. }
$$

Case (C): Mixed-Data, $\sigma_{x z}\left(-L, x_{3}\right)=\bar{\sigma}_{x z}\left(x_{3}\right), u_{x}\left(-L, x_{3}\right)=\bar{u}_{x}\left(x_{3}\right)$

$$
\tilde{u}_{x}(-L)=\frac{1}{H} \int_{-H / 2}^{H / 2} \bar{u}_{x} \mathrm{~d} x_{3}+\frac{\nu(1+\nu)}{E H} \int_{-H / 2}^{H / 2} x_{3} \bar{\sigma}_{x z} \mathrm{~d} x_{3} .
$$

Case (D): Displacement Data, $u_{x}\left(-L, x_{3}\right)=\bar{u}_{x}\left(x_{3}\right), u_{z}\left(-L, x_{3}\right)=\bar{u}_{z}\left(x_{3}\right)$

$$
\left[i i,-\left.\frac{\nu t_{1}^{T} H}{2(1-\nu)} \tilde{u}_{x, 1}\right|_{x_{1}=-L}=\int_{H / 2}^{H / 2}\left\{\sigma_{x x}^{B T}\left(0, x_{3}\right) \bar{u}_{x}+\sigma_{x z}^{B T}\left(0, x_{3}\right) \bar{u}_{z}\right\} \mathrm{d} x_{3} .\right.
$$

We note that $\overline{\boldsymbol{\sigma}}_{x z}$ and $\overline{\boldsymbol{u}}_{z}$, which are quantities normally associated only with transverse plate bending, may have a dominant role in the in-plane extension for case (C) and case (D), respectively.

\section{Transverse Bending}

The governing equation for plate bending may be taken to be

$$
\tilde{u}_{z, 1111}=0 \text {, }
$$

where $\tilde{\boldsymbol{u}}_{z}$ is the mid-plane displacement $\boldsymbol{u}_{z}\left(x_{1}, 0\right)$. At $\boldsymbol{x}_{1}=-\boldsymbol{L}$, the two appropriate 
boundary conditions for plate bending are given below for four canonical sets of boundary data in 3-dimensional elasticity theory:

Case (A): Stress-Data, $\sigma_{x x}\left(-L, x_{3}\right)=\bar{\sigma}_{x x}\left(x_{3}\right), \sigma_{x z}\left(-L, x_{3}\right)=\bar{\sigma}_{x z}\left(x_{3}\right)$

$$
\begin{aligned}
& \tilde{u}_{z, 11}(-L)=-\frac{1}{D} \int_{-H / 2}^{H / 2} x_{3} \bar{\sigma}_{x x} \mathrm{~d} x_{3}, \\
& \tilde{u}_{z, 111}(-L)=-\frac{1}{D} \int_{-H / 2}^{H / 2} \bar{\sigma}_{x z} \mathrm{~d} x_{3} .
\end{aligned}
$$

Case (B): Mixed-Data, $\sigma_{x x}\left(-L, x_{3}\right)=\bar{\sigma}_{x x}\left(x_{3}\right), u_{z}\left(-L, x_{3}\right)=\bar{u}_{z}\left(x_{3}\right)$

$$
\begin{aligned}
\tilde{u}_{z, 11}(-L)= & -\frac{1}{D} \int_{-H / 2}^{H / 2} x_{3} \bar{\sigma}_{x x} \mathrm{~d} x_{3}, \\
\tilde{u}_{z}(-L)= & \frac{6}{H^{3}} \int_{-H / 2}^{H / 2}\left\{\left(\frac{1}{2} H\right)^{2}-x_{3}^{2}\right\} \vec{u}_{z} \mathrm{~d} x_{3} \\
& +\frac{1}{D} \int_{-H / 2}^{H / 2}\left\{\frac{2-\nu}{6(1-\nu)} x_{3}^{3}-\frac{H^{2}}{20} x_{3} \bar{\sigma}_{x x} \mathrm{~d} x_{3} .\right.
\end{aligned}
$$

Case (C): Mixed-Data, $\boldsymbol{\sigma}_{x z}\left(-L, x_{3}\right)=\overline{\boldsymbol{\sigma}}_{x z}\left(x_{3}\right), u_{x}\left(-L, x_{3}\right)=\bar{u}_{x}\left(x_{3}\right)$

$$
\begin{aligned}
\tilde{u}_{z, 111}(-L)= & -\frac{1}{D} \int_{-H / 2}^{H / 2} \bar{\sigma}_{x z} \mathrm{~d} x_{3}, \\
\tilde{u}_{z, 11}(-L)= & -\frac{12}{H^{3}} \int_{-H / 2}^{H / 2} x_{3} \bar{u}_{x} \mathrm{~d} x_{3} \\
& +\frac{1}{D} \int_{-H / 2}^{H / 2}\left\{\frac{(4+\nu) H^{2}}{20(1-\nu)}-\frac{v}{2(1-\nu)} x_{3}^{2} \bar{\sigma}_{x z} \mathrm{~d} x_{3} .\right.
\end{aligned}
$$

Case (D): Displacement-Data, $u_{x}\left(-L, x_{3}\right)=\bar{u}_{x}\left(x_{3}\right), u_{z}\left(-L, x_{3}\right)=\bar{u}_{z}\left(x_{3}\right)$

$$
\begin{aligned}
& {\left[\tilde{u}_{z}+\frac{\nu t_{2}^{F} H^{2}}{8(1-\nu)} \tilde{u}_{z, 11}+\frac{(2-\nu) n_{3}^{F} H^{3}}{48(1-\nu)} \tilde{u}_{z, 111}\right]_{x_{1}=-L}} \\
& \quad=\int_{-H / 2}^{H / 2}\left\{\sigma_{x x}^{B F}\left(0, x_{3}\right) \bar{u}_{x}+\sigma_{x z}^{B F}\left(0, x_{3}\right) \bar{u}_{z}\right\} \mathrm{d} x_{3}, \\
& {\left[\tilde{u}_{z, 1}+\frac{\nu t_{2}^{B} H}{4(1-\nu)} \tilde{u}_{z, 11}+\frac{\left\{6+(2-\nu) n_{3}^{B}\right\} H^{2}}{24(1-\nu)} \tilde{u}_{z, 111}\right] \Lambda_{Y}} \\
& =\int_{-H / 2}^{H / 2}\left\{\sigma_{x x}^{B B}\left(0, x_{3}\right) \bar{u}_{x}+\sigma_{x z}^{B B}\left(0, x_{3}\right) \bar{u}_{z}\right\} \mathrm{d} x_{3} .
\end{aligned}
$$

In the formulae (7.1)-(7.14), $E$ is Young's modulus, $\mathrm{v}$ is Poisson's ratio and $D$ is the flexural rigidity of the plate, defined by

$$
D=\frac{E H^{3}}{12\left(1-\nu^{2}\right)} \text {. }
$$

The constants $\boldsymbol{t}_{1}^{\boldsymbol{T}}, \boldsymbol{t}_{2}^{\boldsymbol{F}}, \boldsymbol{t}_{2}^{\boldsymbol{B}}, \boldsymbol{n}_{\mathbf{3}}^{\boldsymbol{F}}, \boldsymbol{n}_{\mathbf{3}}^{\boldsymbol{B}}$, which depend only on v, are defined by (5.9), (5.10) and are tabulated in Table 1 for $\mathrm{v}=\frac{1}{4}, \frac{1}{3}, \frac{1}{2}$.

When the prescribed edge-data is identically zero, then in cases (A), (B), and (C) the boundary conditions on $\boldsymbol{u}_{\boldsymbol{x}}\left(\boldsymbol{x}_{1}, 0\right)$ or $\boldsymbol{u}_{z}\left(x_{1}, 0\right)$, given by (7.2)-(7.4) and (7.7)-(7.12), 
reduce to simple forms in which the plate thickness $\mathrm{H}$ does not appear. Thus not only do we deduce the correct boundary conditions for the Kirchhoff thin plate theory, but we also observe that in these cases the Kirchhoff solution is in fact the exact interior solution on the mid-plane. (The work of Kolos [9] is concerned with modifying these homogeneous boundary conditions to include (approximately) the effect of edge curvature.) In case (D) however, if we prescribe identically zero displacements, then the boundary conditions (7.5), (7.13), (7.14) certainly do not reduce to the customarily used Kirchhoff boundary conditions, except in the limit $\mathrm{H} \rightarrow 0$. Thus in this case, the Kirchhoff solution would not be the exact interior solution.

More generally, if the edge-data is not identically zero then only those conditions representing resultant forces and couples ((7.2), (7.7), (7.8)) are identical with those of the Kirchhoff theory. In any condition involving displacements, the inhomogeneous terms do not in general simplify any further, even in the limit $\mathrm{H} \rightarrow 0$. These correct forms for displacement boundary conditions are new to the literature, even in the limiting case of the Kirchhoff thin-plate theory.

\section{A clamped infinite plate strip under uniform face pressure distributions}

In all previous sections, we effectively considered only problems with external loads distributed along the edges of the plate. By way of a specific problem, we show in this section that the necessary conditions for a decaying state of plane strain obtained in section (4) are also applicable to plates with other types of loading as well. Moreover, the results for the problem will show that, in this case, classical plate theory significantly underestimates the deflection at the plate center, even for relatively thin plates. It is therefore necessary to retain higher order terms in the outer expansion for an accurate solution at points away from the plate edges.

Consider the same infinite plate strip of section (5) but now with both of its infinitely long edges $\left(x_{1} \equiv L x= \pm L\right)$ fixed and its upper and lower face $\left(x_{3} \equiv H z / 2=\right.$ $\pm H / 2$ ) subject to uniform pressure distributions, $\pm p_{0} / 2$, respectively. Evidently, the plate is in a state of plane strain uniform in the +-direction and we only have to consider a cross section of the plate for some fixed value of $x_{2} \equiv L y$ with

$$
\begin{aligned}
& \sigma_{z z}(x ; \pm 1)= \pm \frac{1}{2} p_{0}, \sigma_{x z}(x ; \pm 1)=0, \quad(|x|<1), \\
& u_{x}( \pm 1, z)=u_{z}( \pm 1, z)=0, \quad(|z|<1)
\end{aligned}
$$

to obtain the elastostatic behaviour of the plate strip.

A particular plane strain field which satisfies (8.1) is given by

$$
\chi_{p}=\frac{p_{0} H^{2}}{32}\left[\frac{x^{2}}{\varepsilon^{2}}\left(3 z-z^{3}\right)+\frac{1}{5} z^{5}\right\rfloor .
$$

The corresponding stress and displacement components are

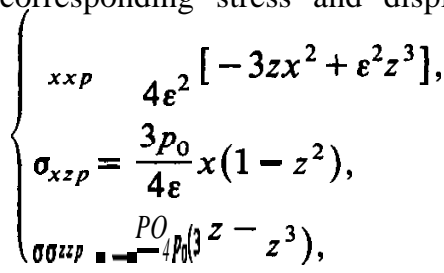


and

$$
\left\{\begin{aligned}
u_{x p}= & \frac{p_{0} H}{16 \varepsilon^{3} G}\left\{-z x\left(x^{2}-2 \varepsilon^{2} z^{2}\right)+\nu x z\left(x^{2}-3 \varepsilon^{2}-\varepsilon^{2} z^{2}\right)\right\} \\
u_{z p}= & \frac{p_{0} H}{64 \varepsilon^{2} G}\left\{\left(x^{4}-12 \varepsilon^{2} x^{2}+6 \varepsilon^{4} z^{2}-\varepsilon^{4} z^{4}\right)\right. \\
& \left.-\nu\left(x^{4}-6 \varepsilon^{2} x^{2}-6 \varepsilon^{2} x^{2} z^{2}+6 \varepsilon^{4} z^{2}+\varepsilon^{4} z^{4}\right)\right\}
\end{aligned}\right.
$$

respectively. By an argument similar to the proof of Theorem (1), the actual plane strain state of the plate strip may be taken in the form

$$
\boldsymbol{u}=\left(\begin{array}{l}
u_{x p} \\
u_{z p}
\end{array}\right)+M u^{V B}+\left(\begin{array}{l}
0 \\
W
\end{array}\right)+\sum_{\mu \in \mathscr{M}} B_{\mu}\left(\boldsymbol{u}_{-\mu}+u_{\mu}\right)
$$

for some unique choice of the coefficients $M, W$ and $\left\{B_{\mu}, \mu \in A^{\prime}\right\}$. Again we regard

$$
u^{(I)}=\left(\begin{array}{l}
u_{x p} \\
u_{z p}
\end{array}\right)+M u^{V B}+\left(\begin{array}{l}
0 \\
W
\end{array}\right)
$$

as the interior solution and the infinite series involving $\left\{u_{-}, \mu \in \mathscr{M}\right\}$ as end corrections. The process of determining the coefficients $\boldsymbol{M}$ and $\bar{W}$ without any reference to the end corrections is similar to that used in section (5); it gives the following expressions for the constants $\boldsymbol{M}$ and $\boldsymbol{W}$ :

$$
\frac{M \varepsilon^{2}}{P 0}=\frac{-4(1-\nu)+6 \nu t_{2}^{B} \varepsilon+4\left[(\nu-2) n_{3}^{B}-3 \nu\right] \varepsilon^{2}+\left[6(1-\nu) t_{2}^{B}-(1+\nu) t_{4}^{B}\right]}{24(1-\mathrm{v})-12 \nu t_{2}^{B} \varepsilon},
$$

$$
\begin{aligned}
\frac{64 \mathrm{G} W \varepsilon^{4}}{P O}= & -(1-\nu)+\left(12-6 \nu t_{2}^{F}-6 \nu\right) \varepsilon^{2}+4(2-\nu) n_{3}^{F} \varepsilon^{3} \\
& +\left[(1+\nu) t_{4}^{F}+6 \nu t_{2}^{F}\right] \varepsilon^{4}-\frac{12 M \varepsilon^{2}}{p_{0}}\left[(1-\nu)+\nu t_{2}^{F} \varepsilon^{2}\right] .
\end{aligned}
$$

The constants $\boldsymbol{t}_{2}^{\boldsymbol{B}}, \boldsymbol{n}_{3}^{\boldsymbol{B}}$, etc., are those defined in section (5) and their numerical values for typical values of $\mathrm{v}$ are given in Table (1). Equations (8.8)-(8.9) are not exact since the non-decaying states induced by the residuals of the end corrections at the opposite edges have been neglected. However, the relative errors in (8.8) and (8.9) are $\mathrm{O}\left(\mathrm{e}^{-2 \gamma / \varepsilon}\right)$ for $\varepsilon \ll 1$, where as in section (5) y $=3.75$... .

The transverse displacement of the center line $\mathrm{z}=0$ of the cross section, as calculated from the interior solution (8.8), with $\boldsymbol{M}$ and $W$ given by (8.8) and (8.9), is

$$
\frac{64 G}{p_{0}} u_{z}^{(I)}(x, 0)=(1-\nu) x^{4}-6(2-\nu) \varepsilon^{2} x^{2}+\frac{12 M \varepsilon^{2}}{-P O}(1-\nu) x^{2}+\frac{64 G}{P O} W,(8.10)
$$

so that in particular

$$
\begin{aligned}
\frac{64 G \varepsilon^{4}}{P 0} u_{2}^{(I)}(0,0)= & \frac{(1-\mathrm{v})+\nu t_{2}^{F} \varepsilon^{2}}{2(1-\nu)-\nu t_{2}^{B} \varepsilon}\left\{4(1-\mathrm{v})-6 \nu t_{2}^{B} \varepsilon+\left[4(2-\nu) n_{3}^{B}+12 \nu\right] \varepsilon^{2}\right. \\
& \left.+\left[(1+\nu) t_{4}^{B}-6(1-\nu) t_{2}^{B}\right] \varepsilon^{3}\right\}-(1-\nu)+\left(12-6 \nu-6 \nu t_{2}^{F}\right) \varepsilon^{2} \\
& +4(2-\nu) n_{3}^{F} \varepsilon^{3}+\left[(1+\nu) t_{4}^{F}+6 \nu t_{2}^{F}\right] \varepsilon^{4} .
\end{aligned}
$$

Since we are neglecting end-effects, there is also a relative error in (8.11) of order 
$\mathrm{O}\left(\mathrm{e}^{-\gamma / \varepsilon}\right)$ for $\varepsilon \ll 1$ However, even if the aspect ratio $\varepsilon$ is as large as $1 / 2$, the error in (8.11) for the displacement of the plate center should still be less than $0.1 \%$.

On the other hand, the classical Kirchhoff plate theory [28] gives

$$
u_{z}^{(P)}(0,0)=\frac{(1-\nu) p_{0}}{64 G \varepsilon^{4}}
$$

which is, as expected, just the leading term of (8.11) as $\varepsilon \rightarrow 0$. We emphasize that the discrepancy between (8.11) and (8.12) is substantial even for relatively small $\boldsymbol{\varepsilon}$ as may be seen from Fig. 5. When the aspect ratio of the plate, $\varepsilon=H / 2 L$, is $1 / 2$ and $\mathrm{v}=\frac{1}{2}$, the classical plate solution for $\boldsymbol{u}_{z}(0,0)$ underestimates the correct value by a factor of 5 . Even when $\varepsilon=\frac{1}{5}$ (and $\boldsymbol{\nu}=0.5$ ), the classical plate solution still underestimates the exact deflection by $40 \%$.

If in (8.11) we retain only terms of order $\boldsymbol{\varepsilon}$ we obtain

$$
u_{z}^{(I)}(0,0)=\frac{(1-\nu) p_{0}}{64 G \varepsilon^{4}}\left[1-\frac{2 \nu t_{2}^{B}}{1-\nu} \varepsilon+\mathrm{O}\left(\varepsilon^{2}\right) \mid\right. \text { ' }
$$

This we would regard as the "first order correction" to the classical plate theory value (8.12). However although (8.13) is indeed a correct asymptotic formula for $\boldsymbol{u}_{z}^{(I)}(0,0)$ as $\varepsilon \rightarrow 0$, over most of the range displayed it gives a worse estimate for $\boldsymbol{u}_{z}^{(I)}(0,0)$ than (8.12); even when $\varepsilon=0.02$ (i.e. $2 L=50 H$ ), (8.13) is not an improvement on (8.12).

This brings into question the value of obtaining all such "first order corrections" to classical plate (or shell) theory (such as in [9]); for in the above case, this first order correction is an improvement on the classical theory only when $\boldsymbol{\varepsilon}$ is so small that the correction is in any case negligible.

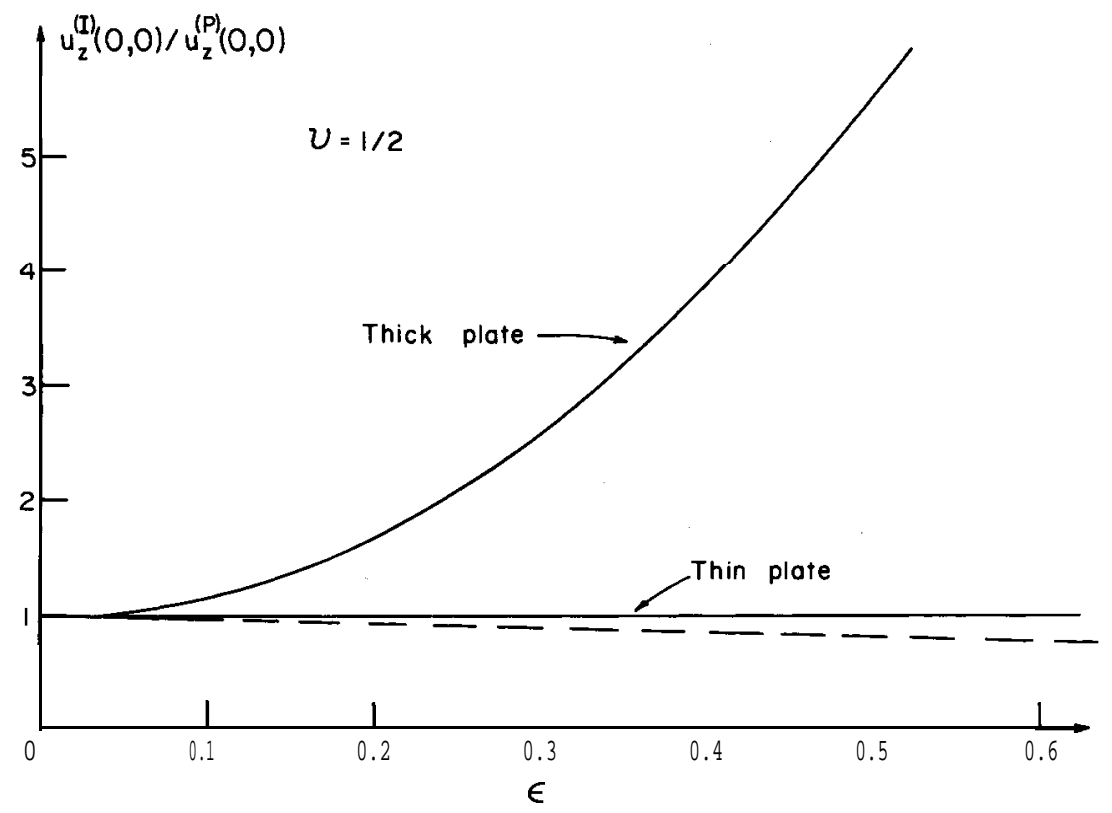

Figure 5. $u_{z}^{(I)}(00) / u_{z}^{(P)}(0,0)$ as a function of $\varepsilon$ for $v=1 / 2$; the dashed curve shows the first order correction to classical plate theory given by $(8.13)$. 


\section{References}

[1] K.O. Friedrichs, The edge effect in the bending of plates, H. Reissner Anniv. Vol., Edwards, Ann Arbor, Mich. (1949) 197-210.

[2] K.O. Friedrichs, Kirchhoff's boundary conditions and the edge effect for elastic plates, Proc. Sym. in Appl Math., Vol. 3, McGraw Hill, New York (1950) 117-124.

[3] K.O. Friedrichs and R.F. Dressler, A boundary layer theory for elastic bending of plates, Comm. Pure Appl. Math. 14 (1961) 1-33.

[4] A.E.H. Love, A Treatise on the Mathematical Theory of Elasticity, 4th ed., Dover, New York, I944.

[5] E.L. Reiss and S. Locke, On the theory of plane stress, Quart. Appl. Math. 19 (1961) 195-203.

[6] E.L. Reiss, Symmetric bending of thick circular plates, J. of S.I.A.M. 10 (1962) 596-609.

[7] A.L. Gol'denveizer, Derivation of an approximate theory of bending of a plate by the method of asymptotic integration of the equations of the theory of elasticity, P.M.M. 26(4) (1962) 668-686.

[8] A.L. Gol'denveizer and A.V. Kolos, On the derivation of two-dimensional equations in the theory of thin elastic plates, P.M.M. 29(1) (1965) 141-155.

[9] A.V. Kolos, Methods of refining the classical theory of bending and extension of plates, P.M.M. 29(4) (1965) 771-781.

[10] A.M.A. Van der Heijden, An asymptotic analysis of a plate with a circular hole, Report No. 581, Jan. 1976, Lab. of Eng. Mech., Delft Univ. of Tech., Delft, The Netherlands.

[11] E.L. Reiss, Extension of an infinite plate with a circular hole, J. of S.I.A.M. 11 (1963) 840-854.

[12] E. Reissner, On the derivation of boundary conditions for plate theory, Proc. Royal Soc., A, 276 (1963) 178-186.

[13] $S$. Nair and E. Reissner, On two-and three-dimensional results for rotationally symmetric deformation of circular cylindrical shells, Int. J. Solids \& Structures 14 (1978) 905-924.

[14] J.P. Benthem, A Laplace transform method for the solution of semi-infinite and finite strip problems in stress analysis, Quart. J. Mech. Appl. Math. 16 (1963) 413-429.

[15] G.D. Gupta, An integral equation approach to the semi-infinite strip problem, J. App. Mech. 40 (1973) 948-954.

[16] F. Erdogan, G.D. Gupta, and T.S. Cook, The numerical solutions of singular integral equations. In: G.C. Sih (ed.), Methods of Analysis and Solutions to Crack Problems, Noordhoff, 1972.

[17] D.B. Bogy, Solution of the plane end problem for a semi-infinite strip, Z.A.M.P. 26 (1975) 749-769.

[18] G.G. Adams and D.B. Bogy, The plane solution for bending of joined dissimilar elastic semi-infinite strips, Int. I. Solids \& Structures 12 (1976) 239-249.

[19] R.D. Gregory and I. Gladwell, The cantilever beam under tension, bending or flexture at infinity, J. of Elasticity 12 (1982) 317-343.

[20] R.D. Gregory, The traction boundary value problem for the elastostatic semi-infinite strip, existence of solutions, and completeness of the Papkovich-Fadle eigenfunctions, J. of Elasticity 10 (1980) 295-327.

[21] M. Gurtin, The Linear Theory of Elasticity. Handbuch der Physik, 6a/3, Springer-Verlag, 1972.

[22] R.D. Gregory, Green's functions, bi-linear forms and the completeness of the eigenfunctions for the elastostatic strip and wedge, J. of Elasticity 9 (1979) 283-309.

[23] E. Sternberg and J.K. Knowles, Minimum energy characterizations of Saint-Venant's solution to the relaxed Saint-Venant Problem, Arch. Rat. Mech. Anal. 21 (1965) 7-107.

[24] M.I. Gusein-Zade, On the conditions of existence of decaying solutions of the two-dimensional problem of the theory of elasticity for a semistrip, P.M.M. 29 (1965) 892-901.

[26] S. Timoshenko and N. Goodier, Theory of Elasticity (2nd ed.), McGraw-Hill, New York, 1951, pp. 35-39.

[27] P. Dixit and D.D. Joseph, The shape of stress free surfaces on a sheared block, S.I.A.M. I. Appl. Math. 42 (1982) 653-677.

[28] S. Timoshenko and W. Woinowsky-Krieger, Theory of Plates and Shells (2nd ed.), McGraw-Hill, New York, 1959.

[29] S. Nair and E. Reissner, Improved upper and lower bounds for deflections of orthotropic cantilever beams, Int. J. Solids Strut. 11 (1975) 961-971.

[30] S. Nair and E. Reissner, On asymptotic expansions and error bounds in the derivation of the two-dimensional shell theory, Studies in Appl. Math. 56 (1977) 189-217. 


\section{Appendix}

\section{St. Venant fields for an infinite strip}

The plane strain St. Venant fields corresponding to the axial extension, bending and flexture by unit tension, bending moment and transverse force, respectively, of the infinite strip $\left|x_{3}\right| \leqslant H / 2,\left|x_{1}\right|<$ oo with traction free sides, are well-known and are quoted here for reference. With $x=x_{1} / L, z=x_{3} /(H / 2)$ and $\varepsilon=H / 2 L$, we have

Unit Tension :

$\sigma^{V T}=\frac{2}{H}\left[\begin{array}{ll}\frac{1}{2} & 0 \\ 0 & 0\end{array}\right], \quad u^{V T}=\frac{L}{2 G H}\left[\begin{array}{l}(1-\nu) x \\ -\nu \epsilon z\end{array}\right]$

Unit Bending:

$\sigma^{V B}=\left(\frac{2}{H}\right)^{2}\left[\begin{array}{cc}-\frac{3}{2} z & 0 \\ 0 & 0\end{array}\right], \quad u^{\nu B}=\frac{3 L^{2}}{G H^{3}}\left[\begin{array}{l}-2(1-\nu) \varepsilon x z \\ (1-\nu) x^{2}+\nu \varepsilon^{2} z^{2}\end{array}\right]$

Unit FIexure:

$\left.\sigma^{V F}=\frac{3}{2 H}\left[\begin{array}{ll}2 z \frac{x}{\varepsilon} & 1-z^{2} \\ 1-z^{2} & 0\end{array}\right], \quad u^{V F=} \frac{L^{3}}{G H^{3}} \mid \begin{array}{l}(1-\nu) 3 \varepsilon x^{2} z+6 \varepsilon^{3} z-(2-\nu) \varepsilon^{3} z^{3} \\ -(1-\nu) x^{3}-3 \nu \varepsilon^{2} x z^{2}\end{array}\right]$

where $\mathrm{G}=E / 2(1+\mathrm{v})$ is the shear modulus.

\section{The Papkovich-F adle eigenfunctions}

The symmetric Papkovich-Fadle eigenfunctions $\left\{\boldsymbol{\sigma}_{\lambda}, \boldsymbol{u}_{\lambda}\right\}$ are those derived from the Airy stress functions

$\chi_{\lambda}=\left[(z-1) \sin \left\{\frac{\lambda}{2}(z+1)\right\}+(z+1) \sin \left\{\frac{\lambda}{2}(z-1)\right\}\right] \mathrm{e}^{-\lambda x / 2 \varepsilon}$

where $\boldsymbol{\lambda}$ is any non-zero root of

$\sin \lambda+\lambda=0$.

The untisymmetric Papkovich-Fadle eigenfunctions $\left\{\boldsymbol{\sigma}_{\mu}, \boldsymbol{\mu}_{\boldsymbol{\mu}}\right\}$ are those derived from the Airy stress functions

$\chi_{\mu}=\left[(z-1) \sin \left\{\frac{\mu}{2}(z+1)\right\}-(z+1) \sin \left\{\frac{\mu}{2}(z-1)\right\}\right] \mathrm{e}^{-\mu x / 2 \varepsilon}$,

where $\mu$ is any non-zero root of

$\sin \mu-\mu=0$.

The non-zero roots of (A2.2) and (A2.4) are all complex and appear in symmetrical sets of four in the complex plane; those roots with a positive real part will be denoted in this paper by

$\mathscr{L}=\{\lambda ; \sin \lambda+\lambda=0, \operatorname{Re}(\lambda)>0\}$

$\mathscr{M}=\{\mu ; \sin \mu-\mu=0, \operatorname{Re}(\mu)>0\}$.

The displacement field $\boldsymbol{u}_{\lambda}$, as derived from (A2.1), is non-unique to within a rigid body motion. For any $\boldsymbol{\lambda}$ in $\mathscr{L}$, we shall make $\boldsymbol{\mu}_{\lambda}$ unique by requiring that $\boldsymbol{\mu}_{\lambda} \rightarrow 0$ as $\mathrm{x} \rightarrow \infty$; then $\boldsymbol{\mu}_{\lambda}=\mathbf{0}\left(\mathrm{e}^{-\boldsymbol{\beta} x / \boldsymbol{e}}\right)$ as $\mathrm{x} \rightarrow 00$ where $2 \boldsymbol{\beta}$ is the smallest real part of the roots of (A2.2) $\left(\boldsymbol{\beta} \simeq 2.106\right.$.. .). Similar remarks apply to $\boldsymbol{\mu}_{\boldsymbol{\mu}}$ for all $\boldsymbol{\mu}$ in $\boldsymbol{M}$.

\section{Axial extension, bending and flexture of a cantilevered semi-infinite strip}

Consider the semi-infinite strip $x_{1} \geqslant 0,\left|x_{3}\right| \leqslant H / 2$ with traction free sides and built -in (cantilevered) at the end $\boldsymbol{x}_{\mathbf{1}}=0$ so that $\boldsymbol{u}=0$. Let the plane strain fields $\left\{\mathrm{a}^{\boldsymbol{B} T}, \boldsymbol{u}^{\boldsymbol{B} T}\right\},\left\{\boldsymbol{\sigma}^{\boldsymbol{B} \boldsymbol{B}}, \boldsymbol{u}^{\boldsymbol{B} \boldsymbol{B}}\right\}$ and $\left\{\boldsymbol{\sigma}^{\boldsymbol{B} \boldsymbol{F}}, \boldsymbol{u}^{\boldsymbol{B} F}\right\}$ be those 
produced in the strip when unit tension, bending moment and transverse force respectively are applied at infinity (in the xi-direction). These fields satisfy the hypotheses of Theorem (1) and so have expansions of the form (2.9), (2.10). In particular, the displacement fields in $\mathrm{x} \equiv x_{1} / L>0$ have the form

$u^{B T}=u^{V T}+\left(\begin{array}{l}U^{B T} \\ 0\end{array}\right)+\sum_{\lambda \in \mathscr{L}} A_{\lambda}^{B T} u_{\lambda}$

$u^{B B}=u^{V B}+\left(\begin{array}{l}0 \\ W^{B B}\end{array}\right)+\Theta^{B B}\left(\begin{array}{c}-z \\ x / \varepsilon\end{array}\right)+\sum_{\mu \in \mathscr{M}} B_{\mu}^{B B} u_{\mu}$,

$u^{B F}=u^{V F}+\left(\begin{array}{l}0 \\ W^{B F}\end{array}\right)+\Theta^{B F}\left(\begin{array}{c}-z \\ x / \varepsilon\end{array}\right)+\sum_{\mu \in \mathscr{\mu}} B_{\mu}^{B F} u_{\mu}$,

where $\boldsymbol{u}^{V T}, \boldsymbol{u}^{V \boldsymbol{B}}$ and $\boldsymbol{u}^{V \boldsymbol{F}}$ are the corresponding St. Venant displacement fields defined in section (1) of this Appendix, and $z \equiv x_{3} /(H / 2)$.

The constants $U^{B T}, W^{B B}, \Theta^{B B}, W^{B F}$ and $\Theta^{B F}$ are uniquely determined from the stress distribution on $x=0$ (by the Corollary to Theorem (2)) which is in turn uniquely determined from the prescribed data. As such, these constants are to be determined as a part of the solution of the relevant boundary value problems. Even without an explicit solution, we have already deduced in Theorem (4) the inequalities $\boldsymbol{U}^{\boldsymbol{B} T} \leqslant 0$, $W^{B F}<0$ and $\Theta^{B B} \leqslant 0$ (with equality holding only if $\mathrm{v}-0$ ). In addition, as a corollary to Theorem (2D) we have deduced the relation

$\Theta^{B F}-\frac{1}{2} H W^{B B}=\frac{3(4+\mathrm{v})}{20 \mathrm{G}}$.

From (A3.4), (3.17), (3.18) we further deduce that

$(2-\nu) n_{3}^{B}+3 \nu\left(\frac{2}{H}\right) t_{2}^{F}=-\frac{6}{5}(1-\nu)$,

where the constants $\boldsymbol{n}_{m}^{\boldsymbol{Y}}, \boldsymbol{t}_{m}^{Y}$ are defined by (5.9), (5.10). The identity (A3.5) is used as a check on numerical computations in [18], Appendix 2. 